Research Article

\title{
Comparison of Simulations of Updraft Mass Fluxes and Their Response to Increasing Aerosol Concentration between a Bin Scheme and a Bulk Scheme in a Deep-Convective Cloud System
}

\author{
Seoung Soo Lee $\mathbb{D}^{1},{ }^{1}$ Chang-Hoon Jung, ${ }^{2}$ Sen Chiao ${ }^{(D)},{ }^{3}$ Junshik Um $\left(\mathbb{D},{ }^{4}\right.$ \\ Yong-Sang Choi, ${ }^{5}$ and Won Jun Choi ${ }^{6}$ \\ ${ }^{1}$ Earth System Science Interdisciplinary Center (ESSIC), University of Maryland, College Park, Maryland, USA \\ ${ }^{2}$ Department of Health Management, Kyungin Women's University, Incheon, Republic of Korea \\ ${ }^{3}$ Center for Applied Atmospheric Research and Education, San Jose State University, San Jose, California, USA \\ ${ }^{4}$ Department of Atmospheric Sciences, Pusan National University, Busan, Republic of Korea \\ ${ }^{5}$ Department of Environmental Science and Engineering, Ewha Womans University, Seoul, Republic of Korea \\ ${ }^{6}$ National Institute of Environmental Research, Incheon, Republic of Korea \\ Correspondence should be addressed to Seoung Soo Lee; cumulss@gmail.com
}

Received 21 December 2018; Revised 19 February 2019; Accepted 12 March 2019; Published 12 June 2019

Academic Editor: Pedro Jiménez-Guerrero

Copyright (c) 2019 Seoung Soo Lee et al. This is an open access article distributed under the Creative Commons Attribution License, which permits unrestricted use, distribution, and reproduction in any medium, provided the original work is properly cited.

\begin{abstract}
Key microphysical processes whose parameterizations have substantial impacts on the simulation of updraft mass fluxes and their response to aerosol are investigated in this study. For this investigation, comparisons of these parameterizations are made between a bin scheme and a bulk scheme. These comparisons show that the differences in the prediction of cloud droplet number concentration (CDNC) between the two schemes determine whether aerosol-induced invigoration of updrafts or convection occurs. While the CDNC prediction leads to aerosol-induced invigoration of updrafts and an associated $20 \%$ increase in the peak value of the updraft-mass-flux vertical profile in the bin scheme, it leads to aerosol-induced suppression of updrafts and an associated 7\% decrease in the peak value in the bulk scheme. The comparison also shows that the differences in ice processes, in particular, in the snow loading lead to the different vertical patterns of the updraft-mass-flux profile, which is represented by the peak value and its altitude, between the schemes. Higher loading of snow leads to around $20-30 \%$ higher mean peak value and its around $40 \%$ higher altitude in the bin scheme than in the bulk scheme. When differences in the CDNC prediction and ice processes are removed, differences in the invigoration and the vertical pattern disappear between the schemes. However, despite this removal, differences in the magnitude of updraft mass fluxes still remain between the schemes. Associated with this, the peak value is around $10 \%$ different between the schemes. Also, after the removal, there are differences in the magnitude between cases with different aerosol concentrations for each scheme. Associated with this, the peak value is also around $10 \%$ different between those cases for each scheme. The differences between the cases with different aerosol concentrations for each scheme are generated by different evaporative cooling and different intensity of gust fronts between those cases. The remaining differences between the schemes are generated by different treatments of collection and sedimentation processes.
\end{abstract}

\section{Introduction}

Recent studies have shown that convective clouds, which include deep convective clouds, are substantially affected by aerosol-cloud interactions and associated feedbacks between increasing aerosol concentration, microphysics, and dynamics $[1-7]$. These deep clouds grow to reach the tropopause and are well-known modulators of global dynamic, hydrological, and energy circulations since these clouds form (1) the cloud regime that regulates a significant portion of global momentum and shortwave and longwave radiation and (2) generates the largest portion of the global precipitation [8-10]. Hence, the interactions between aerosol and clouds and associated 
feedbacks in deep clouds are essential for understanding climate change.

The invigoration of convection is a well-known representation of the feedbacks between increasing aerosol concentration, microphysics, and dynamics in deep clouds $[11,12]$. It has been suggested that an aerosol perturbation (or increasing aerosol concentration) enhances freezing and cloud buoyancy, which leads to the invigoration of convection that accompanies increases in the intensity of updrafts and cloud top heights [11] Figure 1). However, there is a wide variety of reported responses of deep convective clouds to aerosol perturbations in simulations $[3,6,12,15-17]$. For example, some studies have shown strong aerosol-induced invigoration for both cloud systems and single clouds, while others have not even for identical cases $[3,12,17]$. Aerosol-induced invigoration is well known to be dependent on environmental and aerosol conditions $[14,18-20]$. However, each of these identical cases has identical environmental and aerosol conditions. This indicates that the differences in invigoration among simulations are not caused by environmental and aerosol conditions and demonstrates that some modeling frameworks appear to have a propensity to manifest invigoration while others do not $[3,12,17]$. This exemplifies that the simulated feedbacks between increasing aerosol concentration, microphysics, and dynamics are strongly dependent on modeling frameworks and has been raising questions about the credibility of the simulated feedbacks including aerosol-induced invigoration.

As a first step to deal with this credibility issue, we have to identify key cloud processes that create the discrepancy in the simulation of the feedbacks among models. The identification of key processes in turn enables us to identify key quantified causes of the discrepancy and thus acts as a valuable stepping stone to resolve the credibility issue. Motivated by this, as a first step to resolve the credibility issue, this paper focuses on microphysics representations and aims to identify and gain a preliminary understanding of key represented microphysical processes whose different representations play a key role in the discrepancy in the simulation of the feedbacks among models. Note that the identification of the key processes has been rarely performed and the community does not have a clear understanding of those processes themselves [21-24]. The identification of those key processes provides the community with focal processes that the community should pay more attention to and thus enables it to resolve the credibility issue efficiently. Based on this, the identification itself can be considered a valuable effort [21-24]. Hence, this study primarily aims to achieve the identification itself. This means that this study does not take interest in understanding detailed mechanisms through which those identified processes affect the discrepancy in the simulation of the feedbacks.

To fulfill the primary aim, this study compares aerosol influences on tropical deep convection between two frequently used microphysical schemes within a common dynamical framework and common model setup (grid resolution, domain configuration, initial conditions, and forcing). Regarding the selection of the two schemes for the comparison, it is well accepted that there are two types of microphysics schemes, which are bin schemes and bulk schemes [21]. It is believed that there are more substantial or greater differences in the representation of microphysics between these schemes than among bin schemes themselves or bulk schemes themselves since bin schemes and bulk schemes have fundamental differences between them [21, 25-27]. A good example of these fundamental differences is that, in bin schemes, hydrometeor size distributions are explicitly predicted, while in bulk schemes, assumed forms of the distributions are used. This motivates this study to focus on comparisons between a bin scheme and a bulk scheme but not on those between bin schemes or between bulk schemes. For these comparisons, we try to select a representative bin scheme and a representative bulk scheme to draw representative and preliminary, though not general, conclusions from the comparisons. The reality is that comparisons between bin schemes themselves or between bulk schemes themselves or between bin schemes and bulk schemes, in terms of numerous individual microphysical processes, are not established adequately well [21]. Hence, we do not have general statistical information on which bin scheme or which bulk scheme is representative in terms of representation of numerous individual microphysical processes [21]. We also do not have general information on which pair of a bin scheme and a bulk scheme shows representative differences between bin schemes and bulk schemes regarding those representations [21]. Based on this, instead of trying to select perfectly representative schemes, this study relies on rather subjective criteria for the selection of the schemes. The subjective criteria indicate that a selected scheme for bin approach, which is the scheme of Khain and Lynn [28], and a selected scheme for bulk approach, which is the scheme of Thompson and Eidhammer [29], for this study have been implemented into widely used models such as the Advanced Research Weather Research and Forecasting (ARW) model and thus frequently used. Stated differently, the subjective criteria indicate that conclusions from comparisons between these two selected schemes can have some level of broad implications though not perfectly general implications. These subjective criteria follow that in other representative studies, which compare schemes and select two schemes for the comparison, such as the study of Dawson et al. and Morrison et al. [30, 31].

The comparison between the selected schemes enables the isolation of the influences of the representation (or parameterization) of microphysical processes on the discrepancy, which acts as a basis on which the isolation of the key microphysical processes can be performed. A series of numerical simulations is performed in which the impact of the representations of microphysical processes on the discrepancy in the simulations of the feedbacks between increasing aerosol concentration, microphysics, and dynamics is examined. The goal of this study is to identify a set of key microphysical processes whose differences account for most of the discrepancy between the schemes. Note that the representation of the feedbacks in climate models is the primary cause of uncertainties in the prediction of climate changes [32]. The different feedbacks among different 


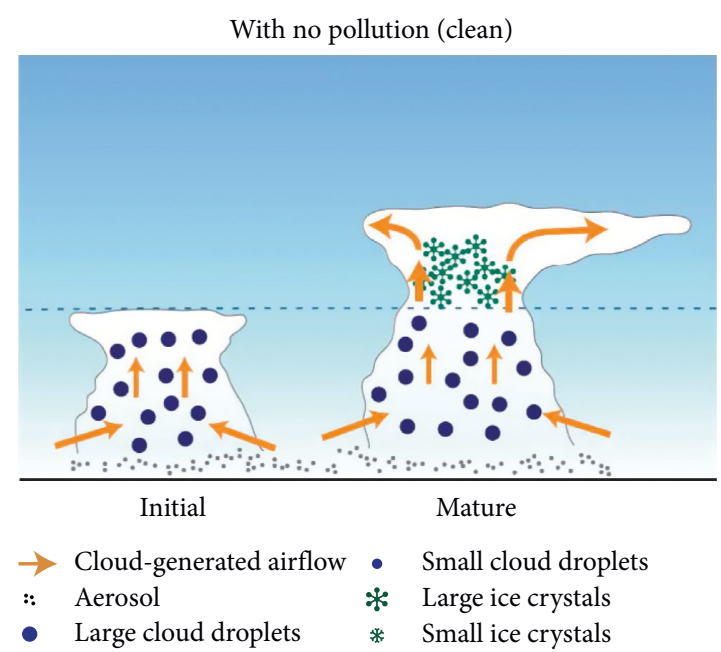

(a)

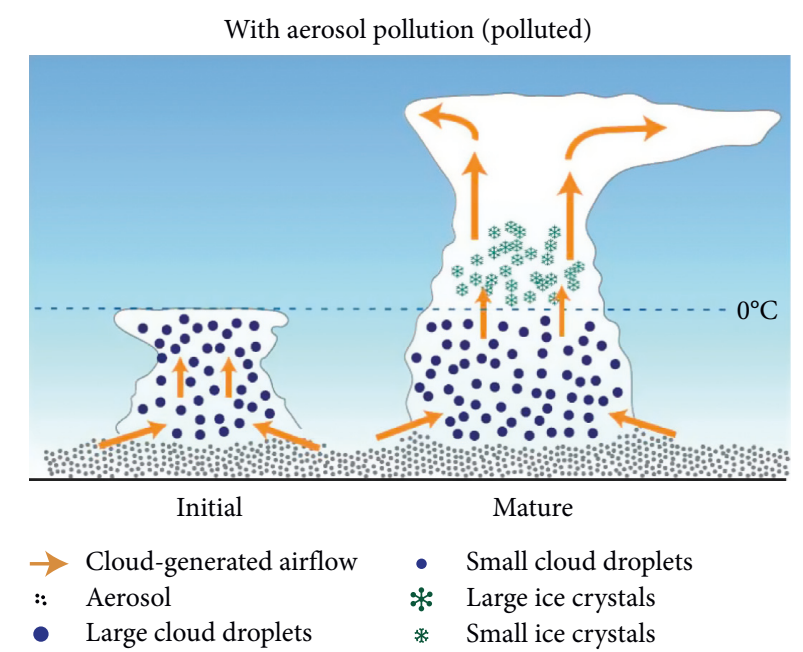

(b)

Figure 1: (a) Airflow generated by convective clouds carries water droplets upwards, leading to the formation of ice crystals in the upper cloud layers. (b) Aerosol pollution (or perturbation) leads to formation of more ice crystals and latent-heat release upon ice crystal formation, which stimulates the updrafts and the vertical growth of clouds (adapted from Lee [13]).

microphysical schemes contribute substantially to those uncertainties by producing a large variation of the feedbacks and their impacts on climate changes among different climate models that are coupled to different microphysical schemes $[12,21,32]$. As a way of reducing this variation as a process of reducing the uncertainties, we need to identify the key microphysical processes whose differences explain most of the variation and develop the schemes in a way that reduces the variation associated with the representation of the key processes. By concentrating on development efforts on these key processes, but not all numerous microphysical processes, the development of the schemes can be performed efficiently.

Accordingly, we aim to find key microphysical processes that collectively remove most of the discrepancy when differences in these processes between the schemes are removed collectively. Hence, in a large portion of simulations in this study, differences in the processes between the schemes are collectively removed. In other words, in a large portion of simulations, differences in two or more potential key processes between the schemes are removed together rather than differences in each of processes removed individually. We do not try to cover the huge parameter space that might need to be explored. Instead, we focus on updrafts, which are represented by updraft mass fluxes, and one of the important indicators of the feedbacks and associated invigoration $[11,12,18]$.

\section{Cloud-System Resolving Model (CSRM)}

The ARW model is employed for this study. The ARW model functions as a CSRM, and this model is not only nonhydrostatic but also compressible. A higher-order scheme, which is developed by Wang et al. [33], is used for the advection of variables, which are particularly related to microphysics. The rapid radiation transfer model (RRTMG), which is described by Mlawer et al. [34] and
Fouquart and Bonnel [35], is utilized to represent radiation processes.

The microphysical processes are represented by two microphysical schemes (or representations). They are the bin scheme of Khain and Lynn [28] and the double-moment bulk microphysics scheme of Thompson and Eidhammer [29]. Here, it should be noted that, in the scheme of Thompson and Eidhammer [29], cloud liquid, snow, and graupel adopt the one-moment prediction of mass-mixing ratio, while cloud ice and rain adopt the two-moment prediction of mass-mixing ratio and number concentration. Henceforth, the former scheme is referred to as "BIN," while the latter scheme is referred to as "BULK." In both of the two microphysics schemes, aerosol mass is prognosed by considering advection, diffusion, and activation-induced removal of the aerosol mass-mixing ratio.

In both microphysics schemes, Köhler theory is applied to droplet activation. When the predicted parcel supersaturation for BIN and the diagnosed parcel supersaturation for BULK are higher than critical supersaturation of aerosol particles, they are assumed to be activated. The aerosol size distribution is assumed to follow the lognormal distributions, and it is assumed that ammonium sulfate comprises aerosol in both of the schemes. The size distribution, which is at the initial time step and for background aerosol, at the altitude of $0.5 \mathrm{~km}$ is depicted in Figure 2. At the other altitudes, background aerosol follows this initial size distribution as well although the initial total concentration of background aerosol varies with altitudes, as exemplified in Figure 3. Figure 3 shows the vertical variation of the initial total concentration of background aerosol which is adopted by this study and for the accumulation mode only. Note that a largest portion of cloud condensation nuclei (CCN) is well known to be included in the accumulation mode when it comes to a general supersaturation regime. In the accumulation mode, the initial background aerosol number concentration in the planetary boundary layer is around $100 \mathrm{~cm}^{-3}$ and it reduces to around 


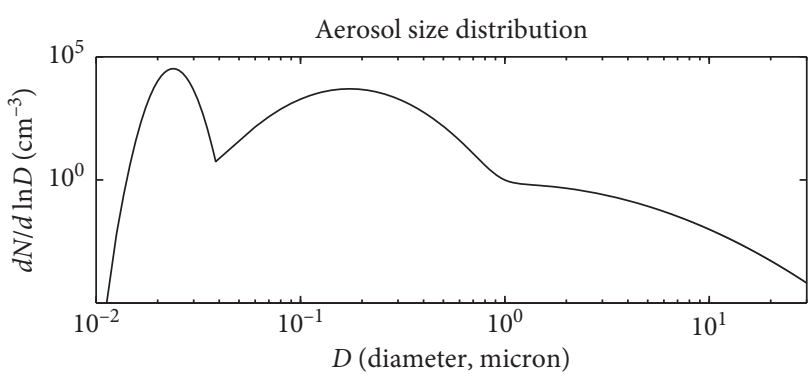

FIGURE 2: Initial aerosol size distribution at the altitude of $0.5 \mathrm{~km}$. $N$ represents the aerosol number concentration per unit volume of air and $D$ the aerosol diameter.

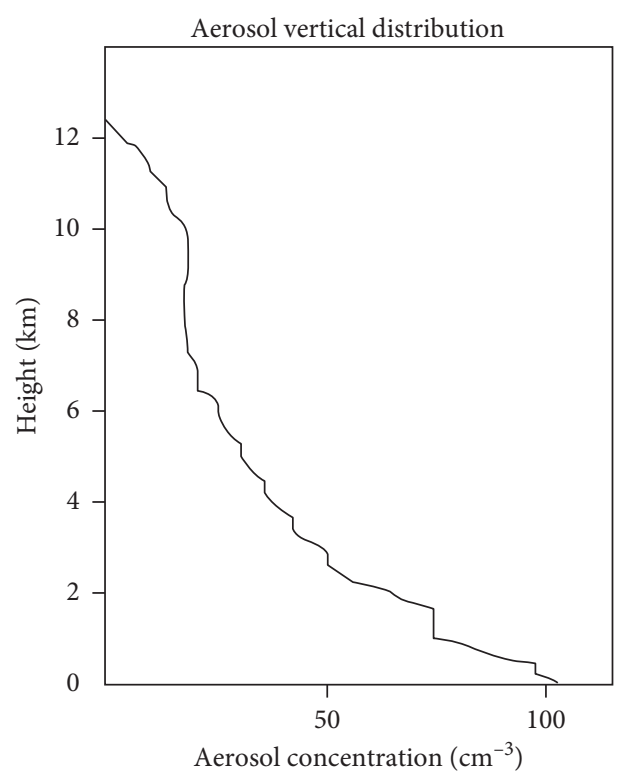

FIGURE 3: Initial vertical distribution of total aerosol concentration in the accumulation mode.

$70 \mathrm{~cm}^{-3}$ at the altitude of $2 \mathrm{~km}$ and then to $20 \mathrm{~cm}^{-3}$ at the altitude of $10 \mathrm{~km}$ (Figure 3). It is assumed that the distribution parameters such as modal diameter and standard deviation of the distribution are fixed over all grid points and the whole simulation period for each mode of the distribution. This means that the form of the distribution in Figure 2 is taken by aerosol over all grid points and the whole period. However, aerosol total number concentration is subject to the spatiotemporal variation since clouds affect aerosol mass as mentioned above. The assumed fixed form of size distribution may overmeasure CCN for nucleation in clouds, which is referred to as secondary nucleation. However, in strong convection in deep clouds here, primary nucleation, which occurs in cloudfree condition, depletes most of $\mathrm{CCN}$; thus, the secondary nucleation plays a negligible role in nucleation, other related microphysical processes, and cloud dynamics (e.g., updraft). This indicates that the weakness from the assumption of the fixed form is not likely to play an important role in results here.

Note that the diffusion of water vapor onto hydrometeors is explicitly calculated by using the predicted supersaturation in BIN. In contrast to this, a saturation adjustment in BULK diagnoses the diffusion by taking water vapor and temperature in the environment into account. While BIN does not assume a specific type of the size distribution of hydrometeors and does make explicit prediction of the distribution, BULK uses basis functions (gamma) with fixed breadth parameters for the size distribution of hydrometeors. In BIN, the size distribution of each hydrometeor class is discretized into 33 size bins and hydrometeor number and mass in each size bin is explicitly predicted. When hydrometeors collide with and collect each other, at each grid point, to represent the collection, collection efficiency for each combination of a size of collecting hydrometeor and that of collected hydrometeor is obtained in BIN. Note that there are 33 times 33 combinations of sizes in BIN, for example, when it comes to collection between two different classes of hydrometeors in BIN. Hence, theoretically, 33 times 33 different collection efficiency values are used for the representation of the collection processes between two different classes of hydrometeors at each grid point in BIN. In BIN, for the representation of sedimentation processes, at each grid point, each terminal velocity, which varies with varying size bin, in each size bin is obtained and hydrometeors in each size bin fall down based on terminal velocity in each bin. Hence, for the representation of sedimentation processes, there are 33 different terminal velocities for each hydrometeor class at each grid point. BULK uses a one-value mass (number) weighted terminal velocity for the sedimentation of hydrometeor mass (number) at each grid point. BULK uses a one-value collection efficiency that is calculated by using the mean volume sizes of collecting and collected hydrometeors at each grid point. In this study, the effect of aerosol on radiation via reflection, scattering, and absorption of radiation by aerosol before its activation is not considered.

\section{Simulation Design}

3.1. Case. An adopted case for this study is an observed mesoscale system. This system involved deep convective clouds. This system was observed between 12:00 LST (local solar time) on January 23th and 12:00 LST on January 25th in 2006. This observation was performed by the TWP-ICE campaign which occurred in Darwin, Australia. The latitude and longitude of the campaign site were $12.47^{\circ} \mathrm{S}$ and $130.85^{\circ} \mathrm{W}$, respectively [36].

3.2. Model Setup. A two-day simulation is performed for the adopted case with BULK, which is named the control-BULK run. For the simulation, the length of the domain in the horizontal direction is set at $120 \mathrm{~km}$ whether it is the eastwest or north-south direction and this length enables the simulation of the mesoscale structure of the system. The length over the vertical direction is $20 \mathrm{~km}$, and this enables us to capture deep convective clouds a portion of which can grow to the tropopause. $1 \mathrm{~km}$ grid spacing in the horizontal direction and a varying grid spacing from approximately $50 \mathrm{~m}$ around the surface to approximately $500 \mathrm{~m}$ around the 
model top in the vertical direction are used to resolve convective cores.

Horizontal boundary conditions are periodic conditions, as described in Fridlind et al. [37]. Observed surface sensible and latent heat fluxes are imposed at the surface. Large-scale forcings of potential temperature and specific humidity as advective tendencies are provided by the TWP-ICE observations. These forcings dictate the net energy and water budget in the domain but not cloud-scale processes. The process of relaxing the horizontal momentum to observed counterpart is applied.

3.3. Additional Runs. The control-BULK run is repeated to look into how aerosol affects updrafts. This sensitivity run is performed by escalating the initial aerosol concentration in the background by a factor of 10 . This run is named "the 10M-BULK run." Then, to examine the sensitivity of updrafts and the effect of aerosol on them to microphysics representations via comparisons between BULK and BIN, we repeat the control-BULK run and the 10M-BULK run by replacing BULK with BIN. These sensitivity runs are named "the control-BIN run" and "the 10M-BIN run." These four simulations constitute "the standard simulations" in this study.

Updrafts are strongly controlled by the buoyancy of a rising air parcel $[9,38]$. The buoyancy is in turn controlled by the loading of hydrometeors, latent-heat processes, and associated microphysical processes $[9,38]$. Based on recent studies such as Rosenfeld et al. [11] and Lee et al. (2013), this paper focuses on latent-heat processes which are associated with not only solid or ice hydrometeors but also liquid hydrometeors. Here, we deal with microphysical processes that are related to overall life cycle of cloud particles: the formation of cloud particles, their growth, interactions between solid particles and liquid particles, which are associated with the invigoration of convection, and their sedimentation to the ground. The classic theory in cloud physics indicates that the formation of cloud particles is affected by water vapor saturation, and byproduct of the formation, which is a starting point of effects of formation on clouds, is cloud particle number concentration such as cloud droplet number concentration (CDNC). The theory also indicates that condensation and deposition, which are a function of water vapor saturation, and autoconversion and accretion between cloud particles govern the growth of cloud particles. As a way of identifying "key cloud processes" which create the discrepancy in the simulations of updrafts and aerosol effects on them between BULK and BIN, we primarily take into account those latent-heat processes, loading of hydrometeors, and microphysical processes.

To see effects of interactions between solid particles and liquid particles on the simulation of updrafts and aerosol effects on them, the standard simulations are repeated with ice processes turned off. These sensitivity runs are named the control-BULK-noice run, the 10M-BULK-noice run, the control-BIN-noice run, and the 10M-BIN-noice run. To see effects of CDNC, these sensitivity simulations are repeated with CDNC fixed at one value over the domain and the simulation period. These additional sensitivity simulations are named the control-BIN-noice-cdnc run, the 10M-BINnoice-cdnc run, the control-BULK-noice-cdnc run, and the $10 \mathrm{M}$-BULK-noice-cdnc run. Effects of the loading of graupel and snow, which explains a large portion of the total loading, are tested by repeating the standard simulations by removing the loading of each of graupel and snow. These sensitivity simulations are named the control-BIN-s run, the 10M-BIN$s$ run, the control-BIN-g run, the $10 \mathrm{M}-\mathrm{BIN}-\mathrm{g}$ run, the control-BULK-g run, the 10M-BULK-g run, the controlBULK-s run, and the 10M-BULK-s run.

We test effects of latent-heat processes related to ice hydrometeors or processes, those to rain evaporation, and those to cloud-liquid evaporation based on recent studies that show importance of those processes (e.g., $[11,17,39,40])$. To test effects of latent-heat processes related to ice hydrometeors, the standard runs with BULK are repeated by turning off ice-related latent-heat processes and these sensitivity runs are named control-BULK-no-ice-lt run and the 10M-BULKno-ice-lt run. To test those effects related to rain evaporation, the control-BIN-noice-cdnc run, the 10M-BIN-noice-cdnc run, the control-BULK-noice-cdnc run, and the 10MBULK-noice-cdnc run are repeated by turning off rain evaporative cooling. These sensitivity simulations are named the control-BIN-no-rain-evp run, the 10M-BIN-no-rain-evp run, the control-BULK-no-rain-evp run, and the 10M-BULKno-rain-evp run. To test those effects related to cloud-liquid evaporation, the sensitivity simulations with rain evaporative cooling turned off are repeated by turning off cloud-liquid evaporative cooling, and these additional sensitivity simulations are named the control-BIN-no-cld-evp run, the 10MBIN-no-cld-evp run, the control-BULK-no-cld-evp run, and the 10M-BULK-no-cld-evp run.

To understand the role played by microphysical processes related to the particle growth in the simulation of updrafts and aerosol effects on them, we test effects of saturation and collection processes (i.e., autoconversion and accretion) on the simulation. To test effects of saturation (collection processes), the control-BIN-no-cld-evp run and the 10M-BIN-no-cld-evp run are repeated by adopting saturation adjustment (collection scheme) in BULK, and these sensitivity simulations are named the control-BIN-sat run and the 10M-BIN-sat run (the control-BIN-col run and the 10M-BIN-col run). To see effects of sedimentation on the simulation of updrafts and aerosol effects on them, the control-BIN-col run and the 10M-BIN-col run are repeated with the sedimentation scheme in BULK, and these sensitivity simulations are named the control-BIN-sed run and the $10 \mathrm{M}-\mathrm{BIN}$-sed run.

Some of the simulations that are described above are performed with multiple microphysical processes which are modified together. For example, in the control-BIN-noicecdnc run, the 10M-BIN-noice-cdnc run, the control-BULKnoice-cdnc run, and the 10M-BULK-noice-cdnc run, both ice processes and CDNC are modified. This disables us from the perfect isolation of effects of ice processes from those of CDNC, or vice versa. Motivated by this, for better isolation of effects of individual microphysical processes, the standard simulations are repeated by modifying a process of interest 
only. These repeated simulations are named by including "only" in their name.

For the isolation of effects of CDNC, the standard simulations are repeated by fixing CDNC, following the method in the "noice-cdnc" runs, yet with ice processes that are turned on. These repeated simulations are the controlBIN-cdnc-only run, the $10 \mathrm{M}-\mathrm{BIN}$-cdnc-only run, the control-BULK-cdnc-only run and the 10M-BULK-cdnconly run. In addition to the "noice-cdnc" runs, it is notable that the above-described simulations to test each of effects of rain and cloud-liquid evaporative cooling, saturation, collection, and sedimentation processes involve other processes which are modified together. For better isolation of each of these processes, the standard simulations are repeated only by turning off rain evaporative cooling, following the same method as in the "no-rain-evp" runs, and then they are repeated again only by turning off cloud-liquid evaporative cooling, following the same method as in the "no-cld-evp" runs. These repeated simulations with rain evaporative cooling off are named the control-BIN-no-rainevp-only run, the 10M-BIN-no-rain-evp-only run, the control-BULK-no-rain-evp-only run, and the 10M-BULKno-rain-evp-only run. These repeated simulations with cloud-liquid evaporative cooling off are named the controlBIN-no-cld-evp-only run, the 10M-BIN-no-cld-evp-only run, the control-BULK-no-cld-evp-only run, and the 10M-BULK-no-cld-evp-only run. For better isolation of roles by the saturation process, the control-BIN run and the $10 \mathrm{M}-\mathrm{BIN}$ run are repeated by adopting saturation adjustment in BULK as in "sat" runs. These simulations are the control-BIN-sat-only run and the 10M-BIN-sat-only run. For better isolation of roles by the collection process, the control-BIN run and the 10M-BIN run are repeated by adopting the collection scheme in BULK as in "col" runs. These simulations are the control-BIN-col-only run and the 10M-BIN-col-only run. Finally, for better isolation of roles by the sedimentation process, the control-BIN run and the $10 \mathrm{M}-\mathrm{BIN}$ run are repeated by adopting the sedimentation scheme in BULK as in "sed" runs. These simulations are the control-BIN-sed-only run and the 10M-BIN-sed-only run.

It should be remembered that, in BULK, among precipitable hydrometeors, snow, and graupel adopt the onemoment prediction, while rain adopts the two-moment prediction. As indicated by Wacker and Seifert [41], Milbrandt and Yau [42], and Milbrandt and McTaggart-Cowan [43], whether the one-moment or the two-moment prediction is used for precipitable hydrometeors affects how the mass of precipitable hydrometeors is distributed in the vertical domain. This can have impacts on results here by altering microphysical factors such as the vertical distributions of loading and latent-heat processes. Motivated by this, the standard simulations with BULK are repeated by replacing the one-moment prediction with the two-moment prediction for snow and graupel. These sensitivity runs are named the control-BULK-2 mt run and the 10M-BULK$2 \mathrm{mt}$ runs. Also, the other BULK simulations are repeated with the two-moment prediction, and these simulations are named by including " $2 \mathrm{mt}$ " in their name. In Table 1 , the description of basic standard and repeated simulations in this study is given. For the sake of brevity of Table 1 and based on the fact that simulations whose name include "only" and " $2 \mathrm{mt}$ " are a simple extension of the basic simulations, these simulations are not included in Table 1 . The description of the simulations in this section and Table 1 is supposed to give their brief outline, and their details are given below in Section 4 .

\section{Results}

In figures below that depict results from the simulations, the simulations with prefixes "control-BULK," "10M-BULK," "control-BIN," and "10M-BIN" in their names are represented by yellow, blue, black, and red lines, respectively. In case, there are two or more simulations whose name is with an identical prefix in a figure, and these simulations use different line types (i.e., solid, dashed, and dotted lines) with an identical line color. The updraft mass fluxes, which are described below, are obtained simply by multiplying the updraft speed with air density. For all the simulations, the updraft speed is predicted. Air density varies negligibly as compared to the variation of updraft speed at each altitude among the simulations; hence, differences in updraft mass fluxes are mostly caused by differences in updraft speed, and contributions by differences in air density to those in updraft mass fluxes are negligible at each altitude among the simulations. This indicates that conclusions drawn based on updraft mass fluxes below are not qualitatively different from those based on updraft speed.

\subsection{Evaluation of the Control-BIN Run and the Control-BULK} Run. To evaluate the control-BIN run and control-BULK run, cloud and precipitation variables that are simulated by the runs are compared to observed counterparts. The cloud fraction, cloud-top height, the liquid-water path (LWP), the ice-water path (IWP), and cumulative precipitation are selected to be those variables. This is based on the fact that the selected variables are well known to be representative variables that are able to give us information on the overall structure of a system of interest [9, 12, 14]. Hence, by selecting the variables, we are able to evaluate how the overall simulation of a system of interest is performed.

The cloud fractions are $0.45(0.48)$ for clouds below $5 \mathrm{~km}$ in altitude, $0.55(0.49)$ for clouds between 5 and $10 \mathrm{~km}$ in altitude, and $0.85(0.78)$ for clouds between 10 and $15 \mathrm{~km}$ in altitude in the control-BIN (control-BULK) run. The observed fractions are 0.49 for clouds below $5 \mathrm{~km}$ in altitude, 0.51 for clouds between 5 and $10 \mathrm{~km}$ in altitude, and 0.82 for clouds between 10 and $15 \mathrm{~km}$ in altitude. The simulated fractions deviate from observation by less than around $10 \%$. The cloudtop height, averaged over the simulation period, is $8.1 \mathrm{~km}$ for the control-BIN run and $7.4 \mathrm{~km}$ for the control-BULK run, and those simulated heights show around 3-5\% discrepancy against an observed height that is $7.8 \mathrm{~km}$. The LWP, averaged over the domain and the simulation period, is $920(734) \mathrm{g} \cdot \mathrm{m}^{-2}$, while the averaged IWP is $85(70) \mathrm{g} \cdot \mathrm{m}^{-2}$ for the control-BIN (control-BULK) run. The observed LWP and IWP are 819 and $77 \mathrm{~g} \cdot \mathrm{m}^{-2}$, respectively, and thus, the differences in LWP and 


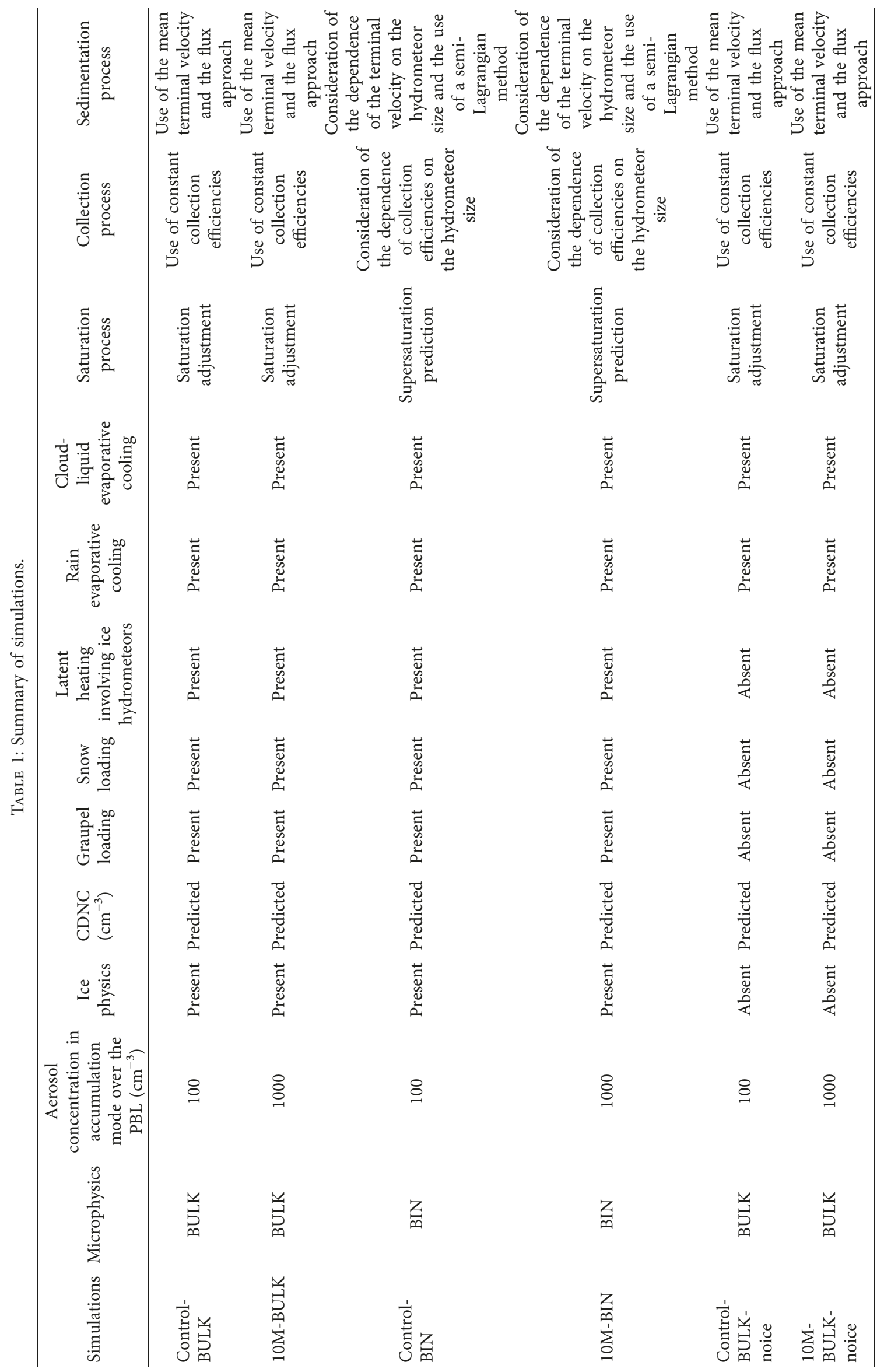




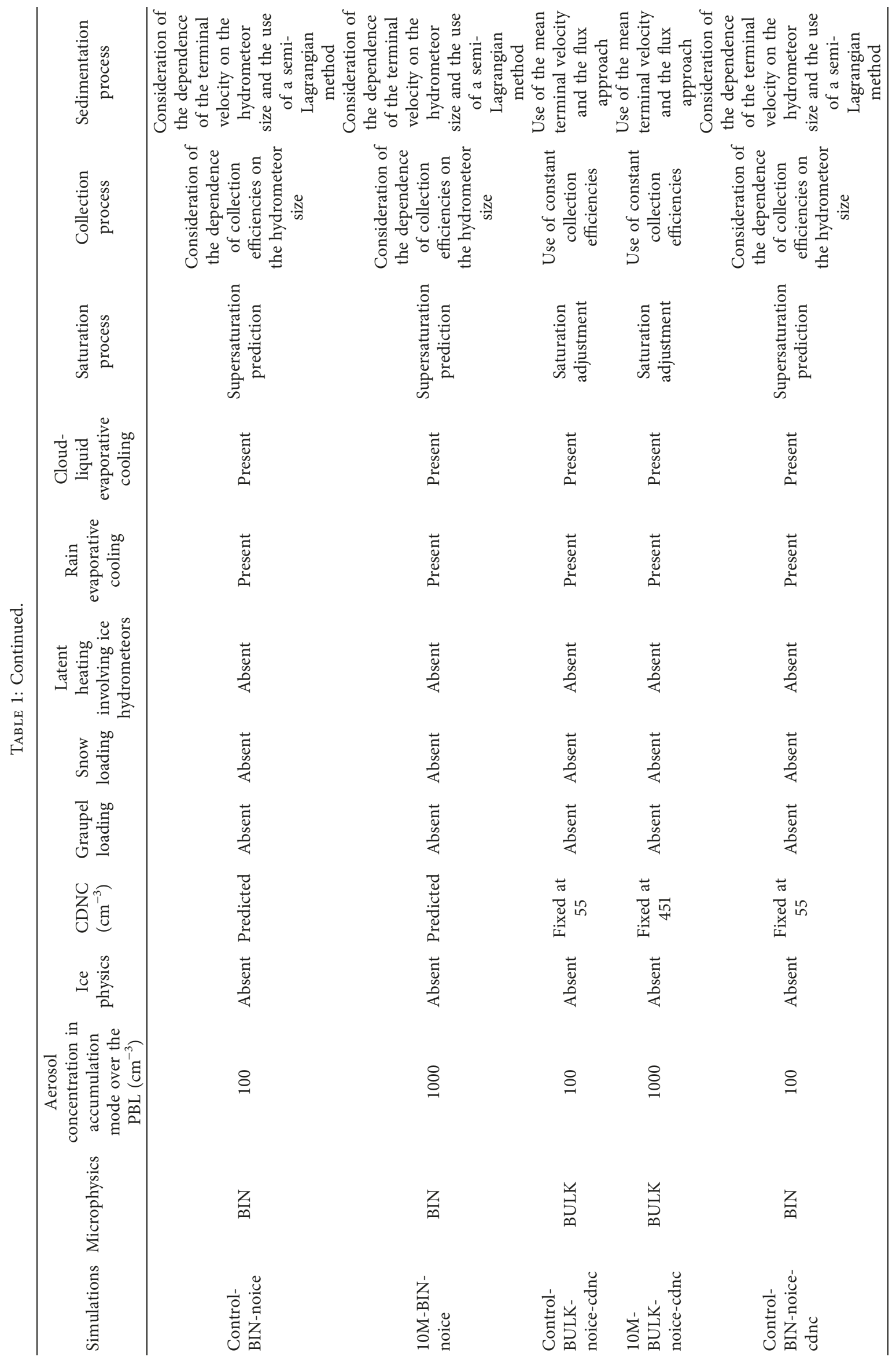




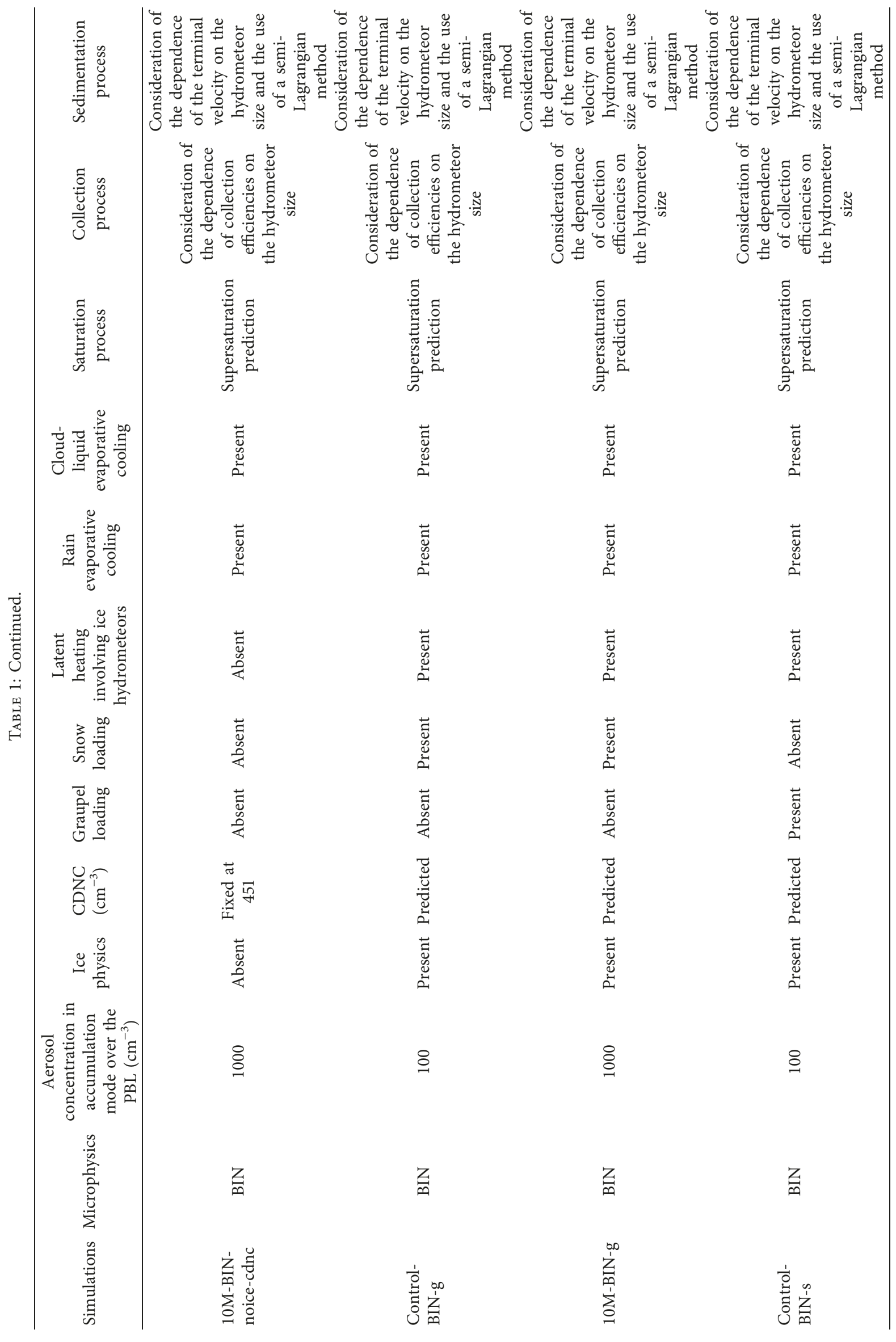




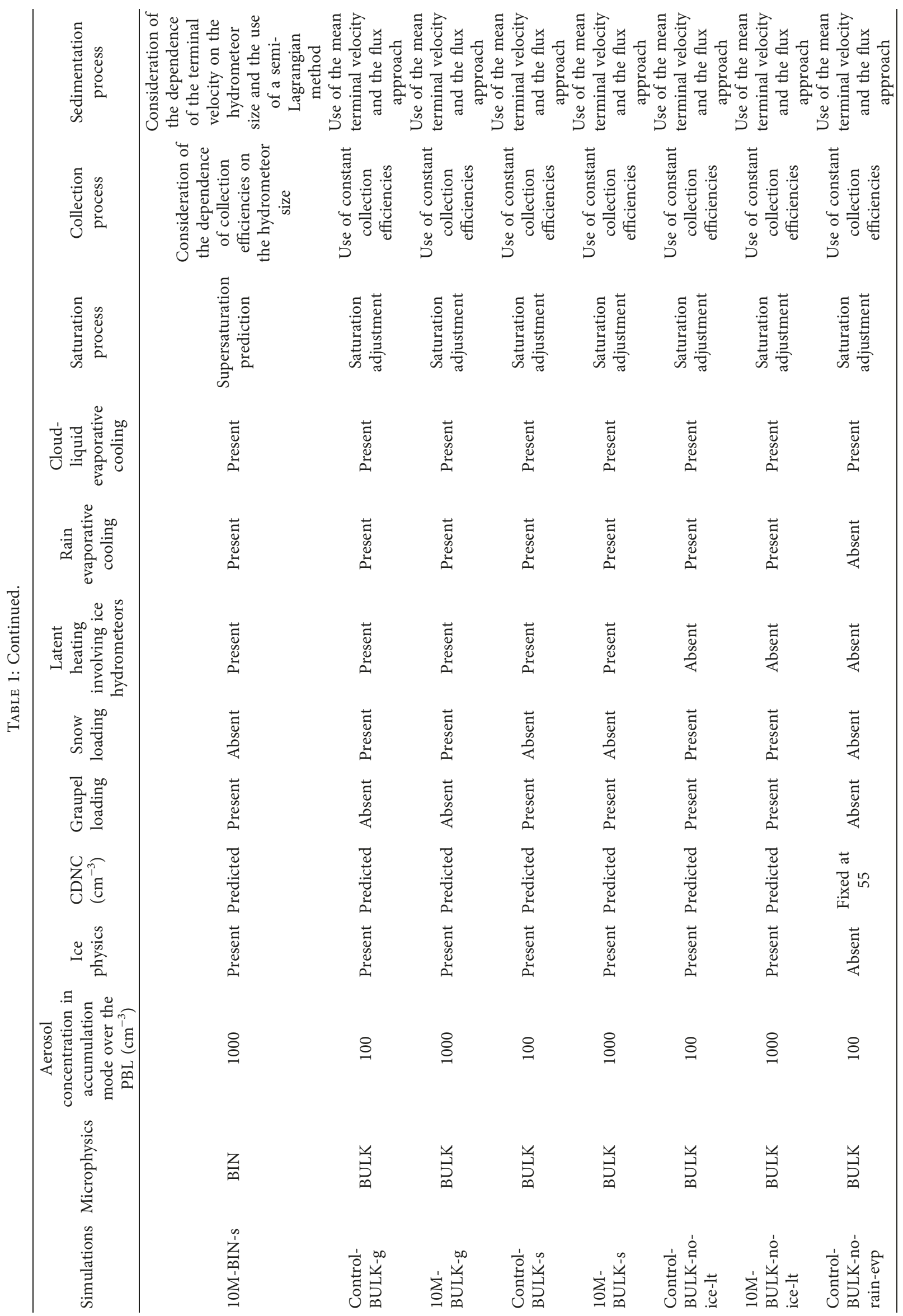




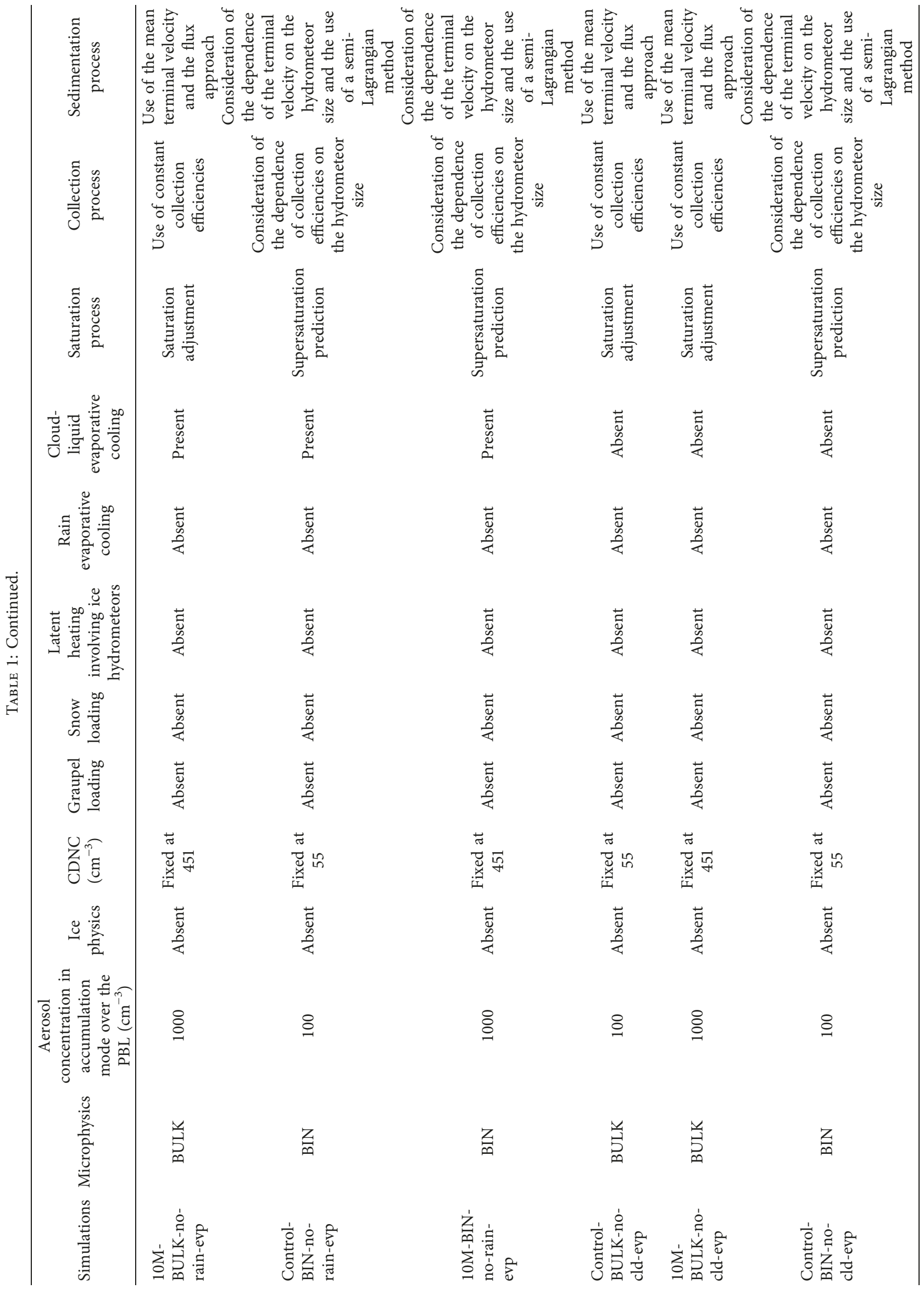




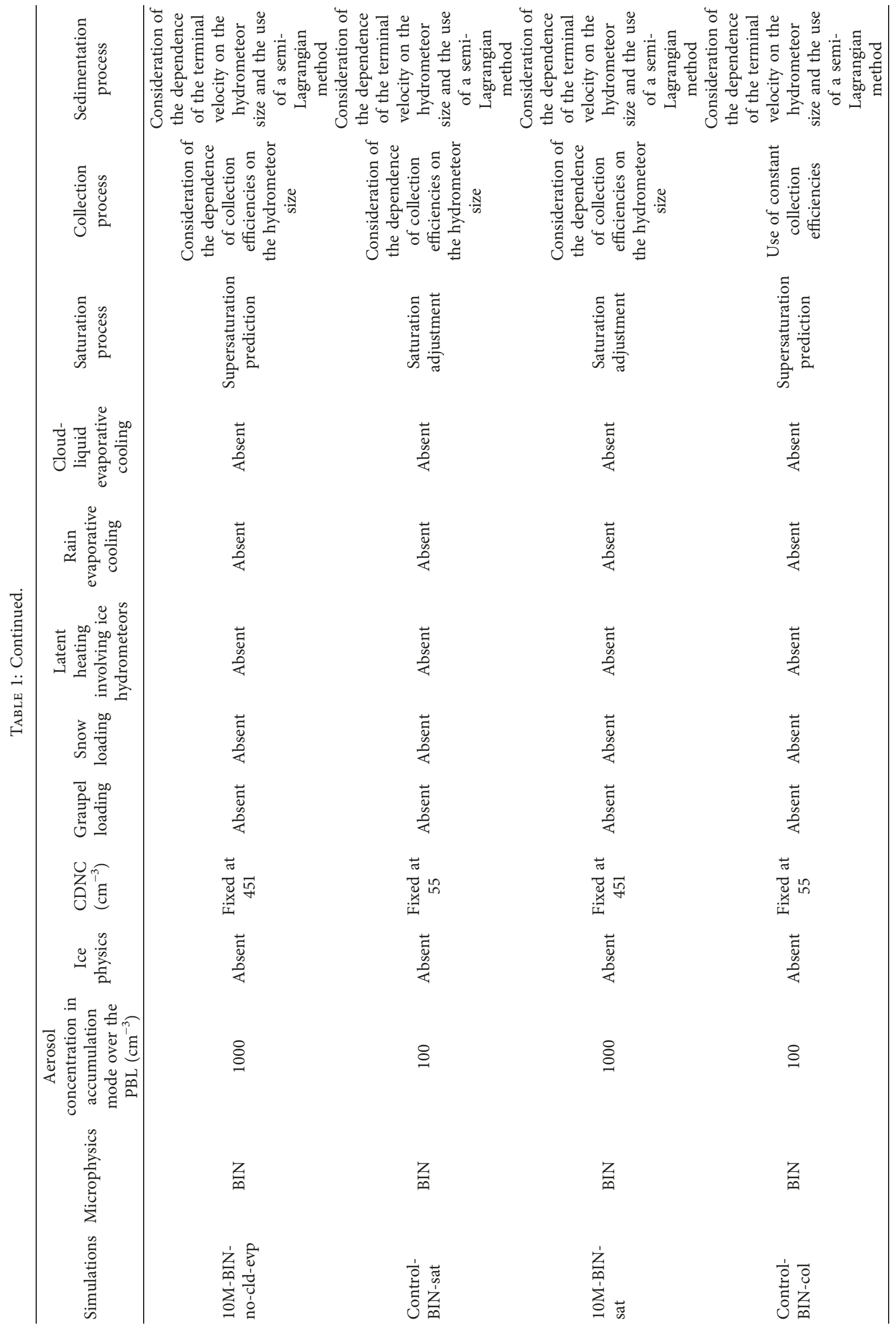




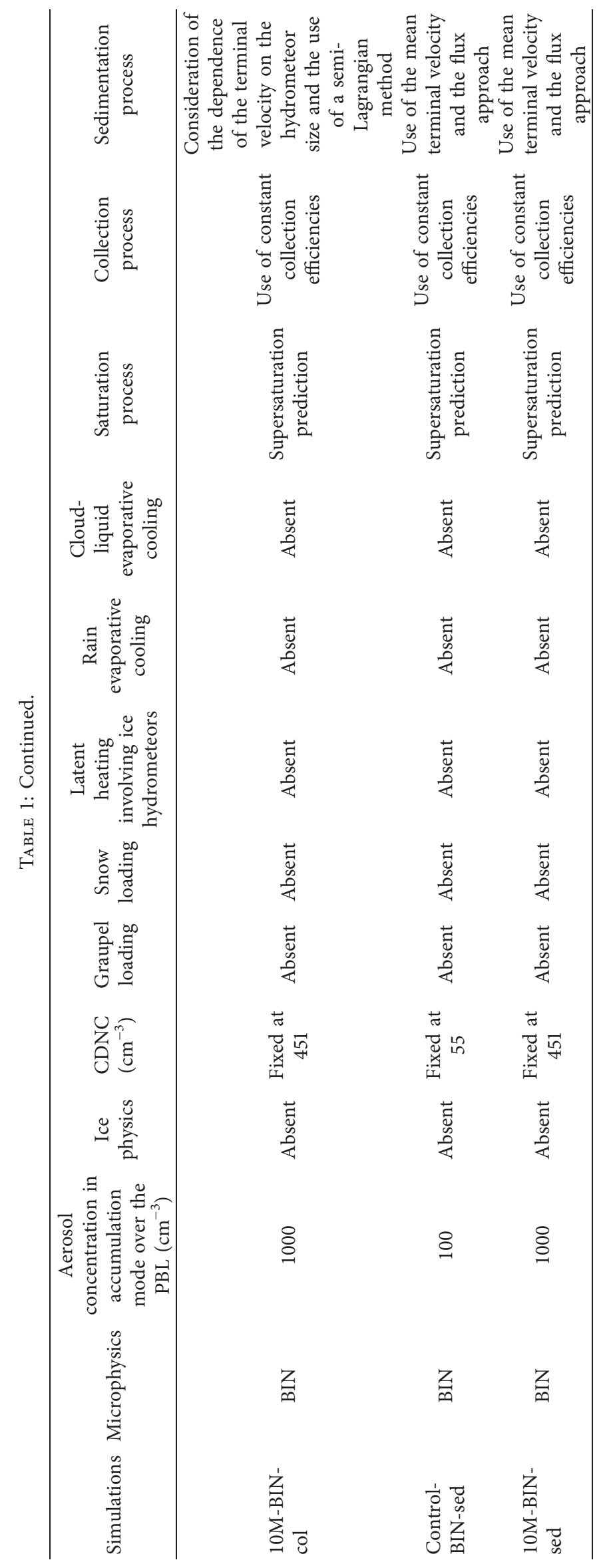


IWP between the control-BIN (control-BULK) run and observation are around $12(10) \%$ and 10 (9)\%. The cumulative precipitation, averaged over the horizontal domain at the last time step, in the control-BIN run (the control-BULK run) is $97.3(98.5) \mathrm{mm}$, which is around 3 (4)\% greater than that for observation that is $94.7 \mathrm{~mm}$. These comparisons provide a fairly good confidence that the simulation of the overall system structure is performed reasonably well. They also show that the averaged fields vary insignificantly from the control-BIN run to the control-BULK.

4.2. Standard Runs with Complete Physics. The controlBULK run, the $10 \mathrm{M}-\mathrm{BULK}$ run, the control-BIN run, and the $10 \mathrm{M}-\mathrm{BIN}$ run, as the standard runs, using standard, "complete" physics are compared in Figure 4. The profiles of updraft mass fluxes vary significantly between simulations with BIN and those with BULK despite the above shown small variation in the averaged fields of cloud fraction, top height, LWP, IWP, and precipitation from the control-BIN run to the control-BULK run.

Profiles of updraft mass fluxes in the control-BULK run show rapid increases up to $2.8 \mathrm{~km}$ and peak at $2.8 \mathrm{~km}$, which are followed by rapid decreases in the fluxes above $2.8 \mathrm{~km}$. The similar rapid increases and decreases are shown in the $10 \mathrm{M}-\mathrm{BULK}$ run (Figure 4). However, the control-BIN run and the 10M-BIN run show less rapid increases in the fluxes to the peak at $3.9 \mathrm{~km}$ and less rapid decreases after the peak, as compared to the simulations with BULK (Figure 4). The magnitude or the value of the peak is from 0.28 to $0.35 \mathrm{~kg} \cdot \mathrm{m}^{-2} \cdot \mathrm{s}^{-1}$ with its mean value of $0.32 \mathrm{~kg} \cdot \mathrm{m}^{-2} \cdot \mathrm{s}^{-1}$ in the simulations with BIN, while it is from 0.25 to $0.27 \mathrm{~kg} \cdot \mathrm{m}^{-2} \cdot \mathrm{s}^{-1}$ with its mean value of $0.26 \mathrm{~kg} \cdot \mathrm{m}^{-2} \cdot \mathrm{s}^{-1}$ in the simulations with BULK. Hence, the altitude of the peak is $39 \%$ higher, and the mean peak value is $23 \%$ higher in the standard BIN runs than in the standard BULK runs (Figure 4).

Another point to make is that the control-BIN run and the 10M-BIN run exhibit an increase in updraft mass fluxes as aerosol concentration increases. Associated with this, the peak value increases by $20 \%$ from 0.28 to $0.35 \mathrm{~kg} \cdot \mathrm{m}^{-2} \cdot \mathrm{s}^{-1}$ with increasing aerosol concentration between the controlBIN run and the 10M-BIN run. However, the control-BULK run and the 10M-BULK run exhibit slight decreases in the flux with increasing aerosol concentration (Figure 4). Associated with this, the peak value decreases by $7 \%$ from 0.27 to $0.25 \mathrm{~kg} \cdot \mathrm{m}^{-2} \cdot \mathrm{s}^{-1}$ with increasing aerosol concentration between the control-BULK run and the 10M-BULK run. In other words, simulations with BIN show aerosol-induced invigoration of convection, while simulations with BULK show aerosol-induced suppression of convection to the contrary. Two frequently used microphysical schemes exhibit different responses to a large aerosol perturbation. To fulfill the aim of this study, instead of trying to understand which microphysics scheme performs better, we focus on the identification of key cloud processes that create the discrepancy among the simulations. In each of the following figures from Figure 5 to Figure 12 which show the profiles of updraft mass fluxes, fluxes in the standard runs are also shown as a reference.

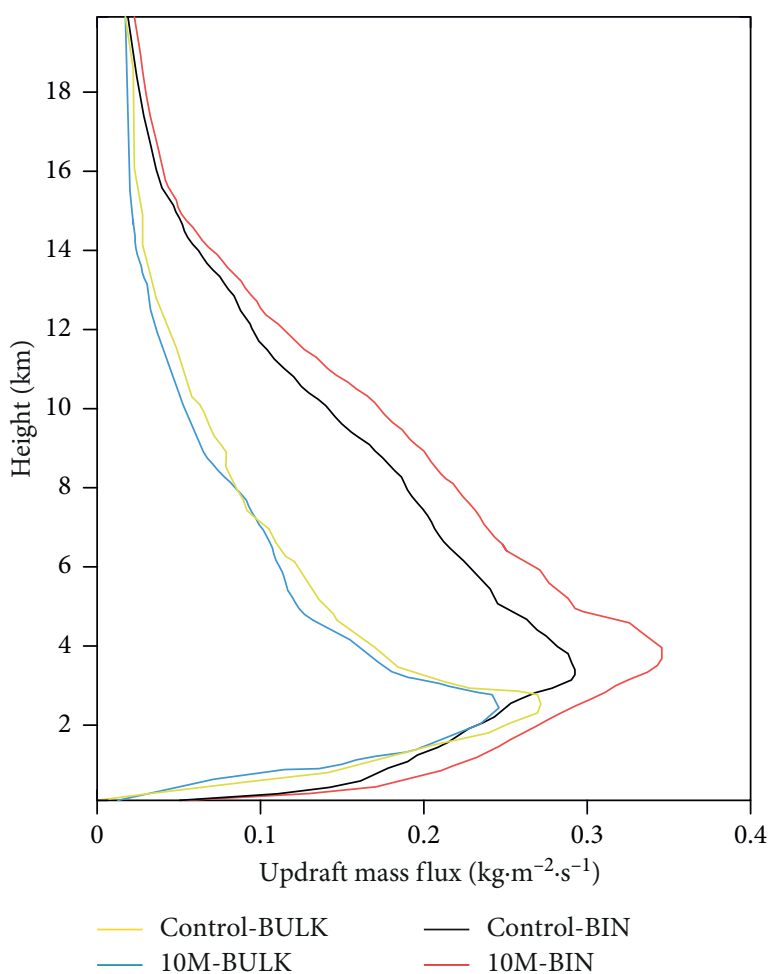

FIGURE 4: Vertical distributions of the time- and domain-averaged updraft mass fluxes for the standard runs (e.g., the control-BULK run, the 10M-BULK run, the control-BIN run, and the 10M-BIN run).

4.3. Ice Processes. Rosenfeld et al. [11] have shown that the suppressed conversion of cloud liquid to rain, which is caused by increasing aerosol concentration, induces increases in cloud liquid and its transportation to places where freezing occurs. This enhances freezing, associated latent heat and buoyancy, and invigorates updrafts and convection. In this invigoration hypothesis, aerosol-induced changes in freezing and buoyancy are a main source of the invigorationrelated changes in updrafts with increasing aerosol concentration. Hence, the different ice processes (involving freezing) may be the main cause of the different responses of updraft mass fluxes to increasing aerosol concentration between the simulations with BULK and those with BIN. Hence, to isolate the effect of ice processes on the different responses among the four standard simulations (i.e., the control-BULK run, the 10M-BULK run, the control-BIN run, and the 10M-BIN run), the four standard simulations are repeated with ice processes turned off completely. In these sensitivity runs, which are the control-BULK-noice run, the 10M-BULK-noice run, the control-BIN-noice run, and the 10M-BIN-noice run, precipitation is formed entirely by warm rain processes (collision and collection of cloud liquid by rain and autoconversion of cloud liquid to rain). In BIN, drops whose size is smaller than $80 \mu \mathrm{m}$ in diameter are classified to be droplets (or cloud liquid), while drops whose size is greater than $80 \mu \mathrm{m}$ in diameter are classified to be rain drops (or rain). Here, autoconversion is a process where droplets collide with and collect each other to form rain. The control-BIN-noice run, the 10M-BIN-noice run, the 
control-BULK-noice run, and the 10M-BULK-noice run show that the differences in the pattern of the vertical variation of updraft mass fluxes (as shown between the standard runs in Figures 4 and 5(a)) nearly disappear (Figures 4 and 5(a)). In this paper, the pattern of the vertical variation of updraft mass fluxes means the altitude of the updraft-mass-flux peak, the peak value, and the rate of changes in updraft mass fluxes with altitudes around the peak. The altitude of the peak is $4.3 \mathrm{~km}$ in the "noice" runs, which is $54 \%$ higher than that in the control-BULK run and the $10 \mathrm{M}$-BULK run and $10 \%$ higher than that in the controlBIN run and the $10 \mathrm{M}-\mathrm{BIN}$ run. The peak value is similar between the "noice" runs since it varies slightly from 0.26 to $0.28 \mathrm{~kg} \cdot \mathrm{m}^{-2} \cdot \mathrm{s}^{-1}$ with the mean peak value of $0.27 \mathrm{~kg} \cdot \mathrm{m}^{-2} \cdot \mathrm{s}^{-1}$ between the runs. This mean peak value is $4(16) \%$ higher (lower) than that in the standard simulations with BULK (BIN).

These sensitivity simulations (as compared to the standard runs in Figures 4 and 5(a)) also show that the fact that simulations with BIN only show aerosol-induced invigoration of convection is robust to whether ice processes are turned off or not although the control-BIN-noice run and the 10M-BIN-noice run show much less aerosolinduced increases in updraft mass fluxes as compared to those in the control-BIN run and in the 10M-BIN run (Figures 4 and 5(a)). These increases are $4 \%$ from 0.27 to $0.28 \mathrm{~kg} \cdot \mathrm{m}^{-2} \cdot \mathrm{s}^{-1}$, which is smaller than $20 \%$ in the control$\mathrm{BIN}$ run and the $10 \mathrm{M}-\mathrm{BIN}$ run. This demonstrates that different ice processes are responsible for the different shapes of the updraft-mass-flux profile but not for the absence of aerosol-induced invigoration with BULK and the presence of the invigoration with BIN.

4.4. CDNC. In addition, it is very likely that there are the different spatiotemporal distributions of CDNCs for an identical background aerosol condition between the controlBIN-noice run and the control-BULK-noice run or between the 10M-BIN-noice run and the 10M-BULK-noice run whose results are depicted in Figure 5(a). With the droplet nucleation, droplets are formed, and CDNC, one of the droplet properties, affects subsequent cloud microphysical and dynamic processes. Since aerosol properties determine CDNC during the nucleation, it is generally considered that CDNC acts as a proxy for aerosol in subsequent cloud processes after the nucleation. Hence, if we want to apply an identical aerosol condition to cloud processes (i.e., both nucleation and subsequent processes after it) in a rigorous manner among different simulations, it is better that not only aerosol condition (before nucleation) but also CDNC distributions are identical between those simulations.

To make sure that the pair of the control-BIN-noice run and the control-BULK-noice run or the pair of the $10 \mathrm{M}$ BIN-noice run and the 10M-BULK-noice run is in an identical aerosol condition for all of cloud processes in a rigorous way, the four sensitivity runs with ice processes turned off are repeated. In these runs, in addition to ice processes turned off, CDNC is fixed at one value for each of aerosol conditions (before nucleation) for the BIN and
BULK runs as a process of removing the differences in the CDNC distributions between the control-BIN-noice run and the control-BULK-noice run or between the 10M-BIN-noice run and the 10M-BULK-noice run. For the control-BINnoice run and control-BULK-noice run with the CDNC fixed, CDNC is fixed at $55 \mathrm{~cm}^{-3}$, while for the $10 \mathrm{M}-\mathrm{BIN}-$ noice run and 10M-BULK-noice run with the CDNC fixed, CDNC is fixed at $451 \mathrm{~cm}^{-3}$. Here, it is seen that about $40-50 \%$ of aerosol particles in the accumulation mode are activated in the "noice" runs. This activation ratio is also applicable to the standard runs whose results are depicted in Figure 4. CDNC is averaged over time and places with nonzero CDNC for each of the runs with ice processes turned off. Then, two averaged CDNCs in the control-BIN-noice (10M-BIN-noice) run and the control-BULK-noice (10MBULK-noice) run are summed and divided by two to obtain the CDNC value that is input to the control-BIN-noice run and the control-BULK-noice run with CDNC fixed (the $10 \mathrm{M}$-BIN-noice run and the 10M-BULK-noice run with CDNC fixed). In each of these sensitivity runs with CDNC fixed, which are the control-BIN-noice-cdnc run, the 10MBIN-noice-cdnc run, the control-BULK-noice-cdnc run, and the 10M-BULK-noice-cdnc run, the obtained CDNC value replaces a predicted $C D N C$ value at each grid point with none-zero predicted CDNC value at each time step.

Comparisons among the simulations with CDNC fixed and ice processes off show that the differences in the pattern of the vertical variation of updraft mass fluxes reduce substantially, as shown in Figure 5(b) in comparisons with those differences between the standard simulations in Figures 4 and 5(b). This is similar to the situation among the control-BIN-noice run, the 10M-BIN-noice run, the control-BULK-noice run, and the 10M-BULK-noice run, as seen in comparisons between Figures 4, 5(a), and 5(b). The altitude of the peak of the updraft-mass-flux vertical profile is similar and at $3.7 \mathrm{~km}$ in the simulations with CDNC fixed and ice processes off, which is $5 \%$ lower than that in the control-BIN run and the $10 \mathrm{M}-\mathrm{BIN}$ run, $32 \%$ higher than that in the control-BULK run and the 10M-BULK run, and $14 \%$ lower than that in the "noice" runs. However, as seen in comparisons between Figures 4 and 5(b), there are still significant differences in the magnitude of updraft mass fluxes among the simulations with CDNC fixed and ice processes off as in the standard runs. Associated with this, the peak value increases by $6 \%$ from $0.32 \mathrm{~kg} \cdot \mathrm{m}^{-2} \cdot \mathrm{s}^{-1}$ in the control-BULK-noice-cdnc run to $0.34 \mathrm{~kg} \cdot \mathrm{m}^{-2} \cdot \mathrm{s}^{-1}$ in the $10 \mathrm{M}-\mathrm{BULK}-$ noice-cdnc run, while it increases by $6 \%$ from $0.36 \mathrm{~kg} \cdot \mathrm{m}^{-2} \cdot \mathrm{s}^{-1}$ in the control-BIN-noice-cdnc run to $0.38 \mathrm{~kg} \cdot \mathrm{m}^{-2} \cdot \mathrm{s}^{-1}$ in the $10 \mathrm{M}-\mathrm{BIN}-$ noice-cdnc run. The mean peak value is $0.33 \mathrm{~kg} \cdot \mathrm{m}^{-2} \cdot \mathrm{s}^{-1}$ between the control-BULKnoice-cdnc run and the $10 \mathrm{M}-\mathrm{BULK}$-noice-cdnc run, while it is $0.37 \mathrm{~kg} \cdot \mathrm{m}^{-2} \cdot \mathrm{s}^{-1}$ between the control-BIN-noice-cdnc run and the 10M-BIN-noice-cdnc run. Hence, when it comes to the mean peak value, the "BIN-noice-cdnc" runs show a $12 \%$ higher value than the "BULK-noice-cdnc" runs. Of interest is that, in "noice-cdnc" runs, both BULK and BIN show the enhancement of updraft mass fluxes as aerosol concentration increases contrary to the situation in the standard simulations (Figures 4 and 5(b)). This demonstrates that 


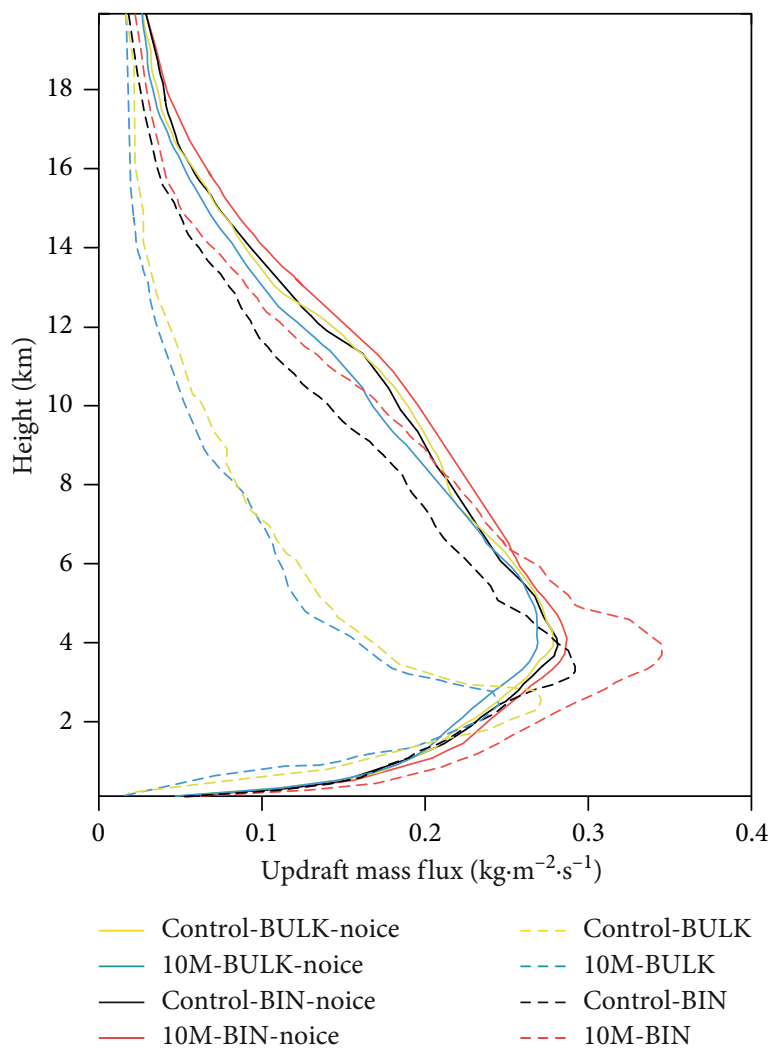

(a)

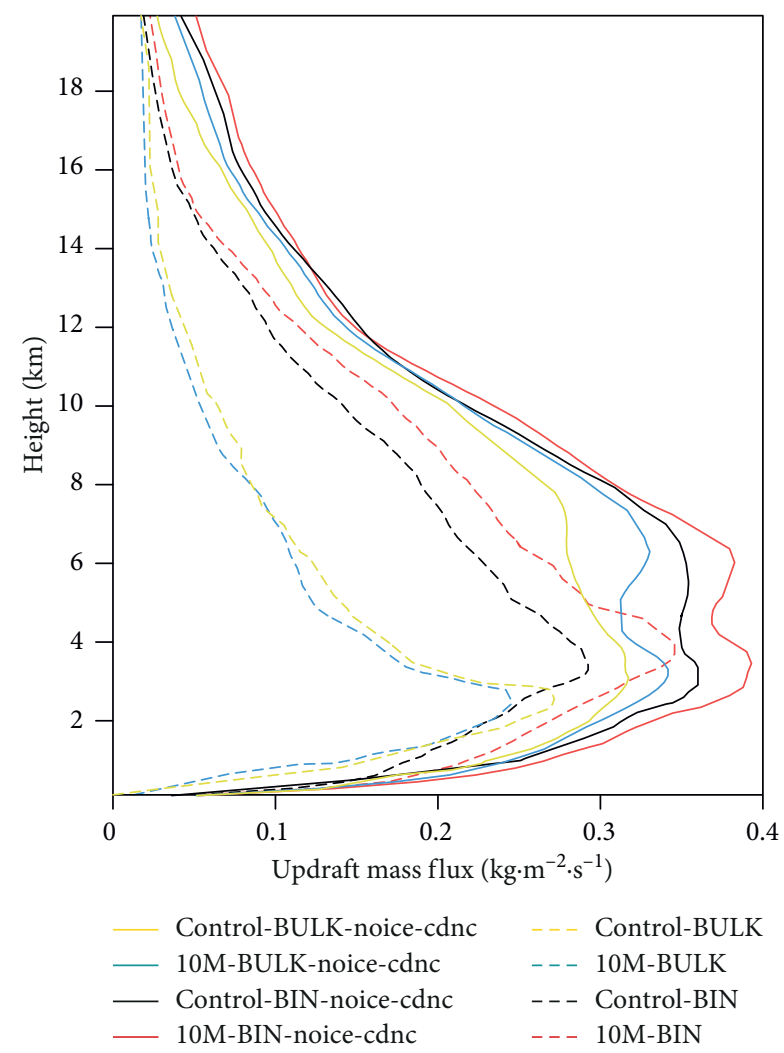

(b)

FiguRE 5: (a) Vertical distributions of the time- and domain-averaged updraft mass fluxes for the standard runs, the control-BULK-noice run, the 10M-BULK-noice run, the control-BIN-noice run, and the 10M-BIN-noice run. (b) Vertical distributions of the time- and domainaveraged updraft mass fluxes for the standard runs, the control-BULK-noice-cdnc run, the 10M-BULK-noice-cdnc run, the control-BINnoice-cdnc run, and the 10M-BIN-noice-cdnc run.

different distributions of CDNC, after nucleation due to different microphysical schemes, for the identical aerosol condition before nucleation between the control-BIN-noice run and the control-BULK-noice run and/or between the $10 \mathrm{M}$-BIN-noice run and the 10M-BULK-noice run are the main cause of the different signs of responses of updraft mass fluxes to increasing aerosol concentration between the two different microphysical schemes. This also demonstrates that how CDNC is predicted after nucleation for an identical aerosol condition before nucleation has a substantial impact on how updraft mass fluxes respond to increasing aerosol concentration.

4.5. Loading of Ice Hydrometeors. The rapid increases and decreases in updraft mass fluxes in the lower atmosphere in the standard simulations with BULK, as shown in Figure 4, may be related to loading effects that are exerted by ice hydrometeors. This is based on the finding that ice processes have an impact on the vertical profile of updraft mass fluxes, as discussed in Section 4.3. We can easily envision that there are much greater loading effects of solid hydrometeors in the standard simulations with BULK which are depicted in Figure 4 than in the standard simulations with BIN which are depicted in Figure 4, particularly around the freezing level that is around $4 \mathrm{~km}$. Remember that these four standard runs are the control-BULK run, the 10M-BULK run, the control-BIN run, and the $10 \mathrm{M}-\mathrm{BIN}$ run. Henceforth, the freezing level indicates an altitude where freezing starts to occur in a rising air parcel, and this altitude is located around $4 \mathrm{~km}$ in this study. The much larger loading or mass of ice hydrometeors around $4 \mathrm{~km}$ can curb the growth of updraft mass fluxes more by inducing greater gravity on the mass that tends to pull down a rising air parcel to the ground or decelerate the parcel more in the standard simulations with BULK than in the standard simulations with BIN. This can lower the peak value in the standard BULK simulations in comparison with the standard BIN simulations. The much larger loading can also "squash" down the vertical profile of updraft mass fluxes around the freezing level in the standard BULK simulations. Note that the profile around the peak between approximately $3 \mathrm{~km}$ and approximately $4 \mathrm{~km}$ in the standard simulations with BULK in Figure 4 appears to be pushed (or squashed) down compared to the profile in the standard simulations with BIN in Figure 4 . The squashed profile accompanies the rapid increases and decreases in updraft mass fluxes around the peak in the standard BULK simulations in Figure 4.

To understand the rapid increases and decreases in updraft mass fluxes in the lower atmosphere in the standard BULK simulations, based on abovementioned conjecture which is related to loading effects, the control-BULK run 
together with the $10 \mathrm{M}-\mathrm{BULK}$ run is repeated by turning off the loading effect of the graupel mass. This is based on the well-known fact that the graupel mass accounts for a significant portion of loading effects particularly in deep clouds. In these sensitivity runs, which are the control-BULK-g run and the 10M-BULK-g run, despite the absence of the loading effect of the graupel mass, graupel particles experience the dynamic, thermodynamic, and microphysical processes as in the standard runs. Comparisons between the control-BULK run, the 10M-BULK run, the control-BULK-g run, and the $10 \mathrm{M}-\mathrm{BULK}-\mathrm{g}$ run indicate that the vertical shape or pattern of the updraft-mass-flux profile does not vary much with the presence or absence of the loading effect of graupel mass (Figure 6(a)). The altitude of the peak value of the profile is $2.8 \mathrm{~km}$ in the control-BULK-g run and the $10 \mathrm{M}-\mathrm{BULK}-\mathrm{g}$ run, which is identical to that in the control-BULK run and the $10 \mathrm{M}-\mathrm{BULK}$ run. The peak value is 0.26 and $0.24 \mathrm{~kg} \cdot \mathrm{m}^{-2} \cdot \mathrm{s}^{-1}$ in the control-BULK-g run and the 10M-BULK-g run, respectively, which shows a slightly $4 \%$ lower value than that in the control-BULK run and the 10M-BULK run, respectively. Then, the control-BULK run and the 10M-BULK run are repeated with the absence of the snow loading, based on the fact that snow and graupel accounts for a significant portion of the loading effect. In these sensitivity runs, which are the control-BULK-s run and the 10M-BULK-s run, despite the absence of the loading effect of the snow mass, snow particles experience the dynamic, thermodynamic, and microphysical processes as in the standard runs. The control-BULK run, the $10 \mathrm{M}-\mathrm{BULK}$ run, the control-BULK-s run, and the $10 \mathrm{M}-$ BULK-s run are compared, and this comparison shows significant changes in the vertical shape of updraft mass fluxes due to the presence or the absence of the snow loading in the simulations with BULK, although the qualitative nature of responses of updraft mass fluxes to increases in aerosol concentration does not vary with whether the snow loading is considered or not (Figure 6(a)). By removing the snow loading in the control-BULK-s run and the 10M-BULK-s run, the altitude and magnitude of the updraft-mass-flux peak and the increases and decreases of the fluxes around the peak in these sensitivity runs become similar to those in the controlBIN run and the 10M-BIN run (Figure 6(a)). Associated with this, the altitude of the peak value increases by $39 \%$ from $2.8 \mathrm{~km}$ in the control-BULK run and the $10 \mathrm{M}-\mathrm{BULK}$ run to $3.9 \mathrm{~km}$ in the control-BULK-s run and the $10 \mathrm{M}-\mathrm{BULK}-\mathrm{s}$ run, while the peak value increases by $22(28) \%$ from $0.27(0.25)$ $\mathrm{kg} \cdot \mathrm{m}^{-2} \cdot \mathrm{s}^{-1}$ in the control-BULK (10M-BULK) run to 0.33 $(0.32) \mathrm{kg} \cdot \mathrm{m}^{-2} \cdot \mathrm{s}^{-1}$ in the control-BULK-s (10M-BULK-s) run.

The vertical distribution of the mass density of snow for the 10M-BULK run and the 10M-BIN run is shown in Figure 6(b). There is much higher mass density of snow in the $10 \mathrm{M}-\mathrm{BULK}$ run than in the $10 \mathrm{M}-\mathrm{BIN}$ run, in particular, around the freezing level. This explains the much larger loading of snow in the standard simulations with BULK than in the standard simulations with BIN, which lowers the value of the updraft-mass-flux peak and pushes down the updraftmass-flux profile around $3 \mathrm{~km}$ and around $4 \mathrm{~km}$ in the standard simulations with BULK as demonstrated by the sensitivity simulations with the loading of snow turned off in Figure 6(a).
To see effects of the loading of snow and graupel for BIN, the control-BIN run and the 10M-BIN run are repeated by removing snow loading and graupel loading, respectively. These sensitivity runs are the control-BIN-s run, the $10 \mathrm{M}-$ BIN-s run, the control-BIN-g run, and the 10M-BIN-g run. As seen in Figure 6(c), whether snow loading or graupel loading is turned off, both the response of updraft mass fluxes to increasing aerosol concentration and the pattern of the vertical variation of updraft mass fluxes do not change significantly in the simulations with BIN. Associated with this, the altitude and magnitude of the peak varies less than $6 \%$ from the standard BIN runs to the BIN runs with either snow loading removed or graupel loading removed.

4.6. Latent Heating and Cooling of Ice Hydrometeors. To test roles played by latent heating and cooling related to ice processes and aerosol effects on them in the shapes of the updraft-mass-flux vertical profile in the control-BULK run and the 10M-BULK run, relative to those played by the loading effect of snow and aerosol influences on it, the control-BULK run and the 10M-BULK run are repeated by turning off the effect of latent heating and cooling from freezing, melting, deposition, and sublimation. These sensitivity simulations are the control-BULK-no-ice-lt run and the 10M-BULK-no-ice-lt run. Note that in the controlBULK-noice run and the 10M-BULK-noice run, as depicted in Figure 5(a), ice hydrometeors are absent or the mass of ice hydrometeors is zero; thus, both loading and latent heating or cooling related to ice hydrometeors are absent. However, in the control-BULK-no-ice-lt run and the 10MBULK-no-ice-lt run, the effect of latent heating related to ice hydrometeors on temperature is only turned off and ice processes are allowed to generate ice hydrometeors. Therefore, the loading of ice hydrometeors is present in these runs. They show results whose qualitative nature is similar to that in the control-BULK run and the 10M-BULK run when it comes to the shape of the vertical profile of updraft mass fluxes and updraft-mass-flux responses to increasing aerosol concentration, as shown in Figure 6(d). Associated with this, the altitude of the updraft-mass-flux peak increase slightly only by 7 (4)\% from $2.8 \mathrm{~km}$ in the control-BULK (10MBULK) run to $3.0(2.9) \mathrm{km}$ in the control-BULK-no-ice-lt (10M-BULK-no-ice-lt) run. The peak value also increases slightly only by $4 \%$ from $0.27(0.25) \mathrm{kg} \cdot \mathrm{m}^{-2} \cdot \mathrm{s}^{-1}$ in the control-BULK (10M-BULK) run to $0.28(0.26) \mathrm{kg} \cdot \mathrm{m}^{-2} \cdot \mathrm{s}^{-1}$ in the control-BULK-no-ice-lt (10M-BULK-no-ice-lt) run. Hence, the effect of latent heating and cooling (related to ice processes) on the shapes of the updraft-mass-flux vertical profile in the simulations with BULK is negligible in comparison with the effect of the snow loading.

Of interest is that, as seen in Figure 6(d), there is a slight increase in updraft mass flux in the control-BULK-no-ice-lt run, as compared to the control-BULK run, and in the 10MBULK-no-ice-lt run, as compared to the 10M-BULK run. This is despite ice-process-related latent heating which enhances buoyancy in updrafts and is turned off in the control-BULK-no-ice-lt run and the 10M-BULK-no-ice-lt run. Increases in ice-process-related latent heating enhance 

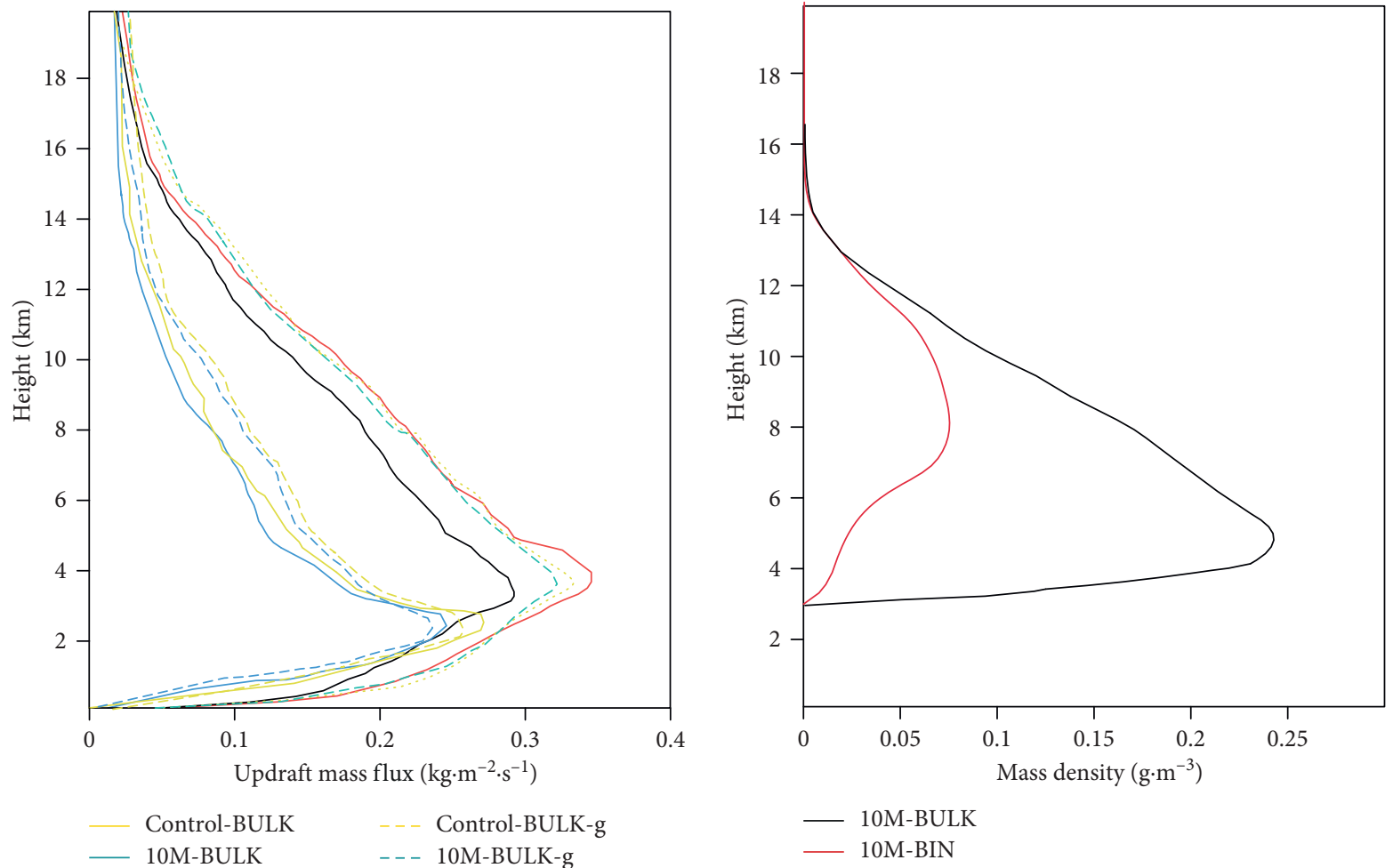

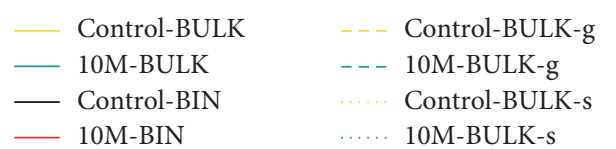

(a)

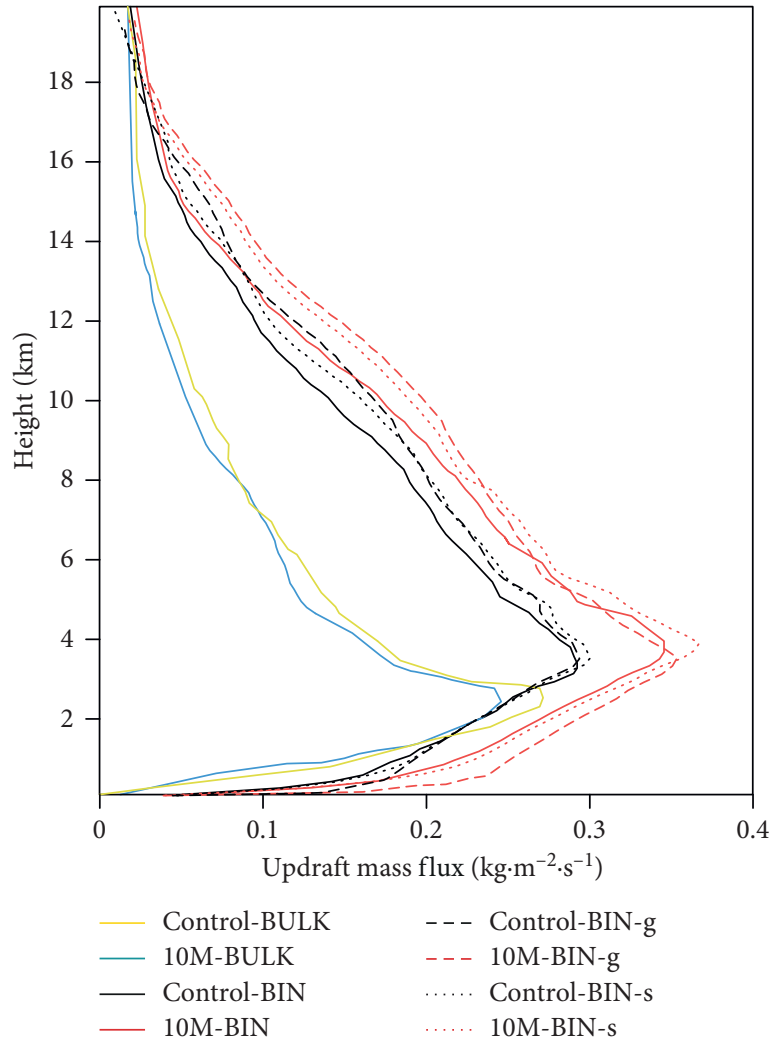

(c) (b)

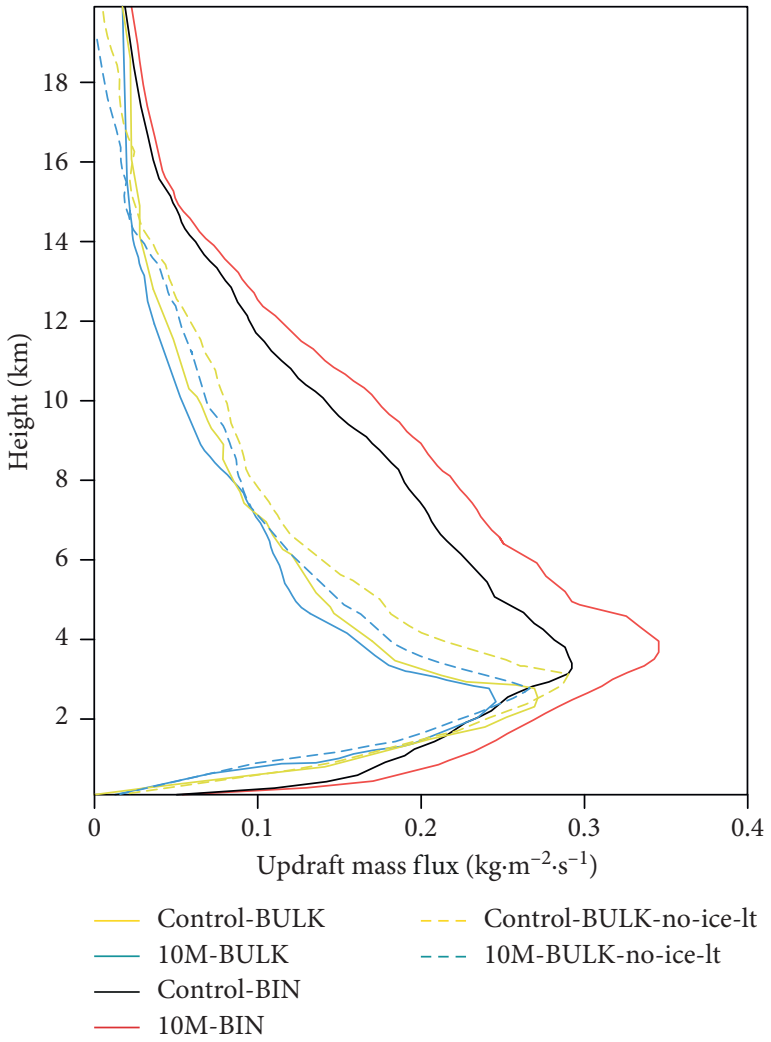

(d)

FIgURE 6: (a) Vertical distributions of the time- and domain-averaged updraft mass fluxes for the standard runs, the control-BULK-g run, the $10 \mathrm{M}-\mathrm{BULK}$-g run, the control-BULK-s run, and the 10M-BULK-s run. (b) Vertical distributions of the time-and domain-averaged snow mass density for the 10M-BULK run and the 10M-BIN run. (c) Vertical distributions of the time- and domain-averaged updraft mass fluxes for the standard runs, the control-BIN-g run, the $10 \mathrm{M}-\mathrm{BIN}-\mathrm{g}$ run, the control-BIN-s run, and the $10 \mathrm{M}-\mathrm{BIN}-\mathrm{s}$ run. (d) Vertical distributions of the time- and domain-averaged updraft mass fluxes for the standard runs, the control-BULK-no-ice-lt run, and the 10M-BULK-no-ice-lt run. 
buoyancy and updraft speed. These increases in updraft speed in turn increase the mass of ice-phase hydrometeors and thus the loading of ice-phase hydrometeors by enhancing supersaturation and deposition. These increases in loading tend to reduce buoyancy and updraft speed. When ice-process-related latent heating is turned off as in the control-BULK-no-ice-lt run and the 10M-BULK-no-ice-lt run, not only ice-process-related latent heating is removed but also the associated increases in the loading of ice-phase hydrometeors are reduced. Effects of reduction in the loading outweighs effects of the removal of ice-processrelated latent heating, resulting in a slight increase in the updraft speed as in the control-BULK-no-ice-lt and the 10M-BULK-no-ice-lt (Figure 6(d)). In other words, there is competition between effects of reduction in the loading and effects of the removal of ice-process-related latent heating in the control-BULK-no-ice-lt and the 10M-BULK-no-ice-lt run. This competition leads to just the slight change in the updraft mass flux in the control-BULK-no-ice-lt, as compared to the control-BULK run, and in the 10M-BULK-noice-lt run, as compared to the 10M-BULK run (Figure 6(d)).

Here, it is notable that snow gains its mass mainly through other processes such as the riming of liquid hydrometeors onto snow than deposition. The time- and domain-averaged rate of the riming of liquid hydrometeors onto snow is $8.391,8.402,8.261$, and $8.333 \times 10^{-2} \mathrm{~g} \cdot \mathrm{m}^{-2} \cdot \mathrm{h}^{-1}$, while that of deposition is $1.011,1.002,0.582$, and $0.551 \times 10^{-2} \mathrm{~g} \cdot \mathrm{m}^{-2} \cdot \mathrm{h}^{-1}$ in the control-BULK run, the $10 \mathrm{M}-$ BULK run, the control-BULK-no-ice-lt run, and the $10 \mathrm{M}-$ BULK-no-ice-lt run, respectively. Here, we see that the riming rate is around one order of magnitude greater than the deposition rate, and the riming rate shows insignificant change around $1 \%$ or less changes between the controlBULK run and the control-BULK-no-ice-lt run or between the 10M-BULK run and the 10M-BULK-no-ice-lt run. Due to this, despite substantial around $40-45 \%$ changes in the deposition rates between the control-BULK run and the control-BULK-no-ice-lt run or between the 10M-BULK run and the 10M-BULK-no-ice-lt run, the snow loading does not change significantly in the control-BULK-no-ice-lt run, as compared to the control-BULK run, and in the 10M-BULKno-ice-lt run, as compared to the 10M-BULK run. The snow loading, which is defined to be the time- and domainaveraged snow mass density, is $0.147,0.152,0.139$, and $0.144 \mathrm{~g} \cdot \mathrm{m}^{-3}$ in the control-BULK run, the $10 \mathrm{M}-\mathrm{BULK}$ run, the control-BULK-no-ice-lt run, and the 10M-BULK-noice-lt run, respectively. This leads to the negligible changes in the shapes of the updraft-mass-flux vertical profile in the control-BULK-no-ice-lt run, as compared to the controlBULK run, and in the 10M-BULK-no-ice-lt run, as compared to the 10M-BULK run (Figure 6(d)).

4.7. Cooling from Rain Evaporation. It has been known that changes in rain evaporation, which are induced by aerosol, can cause those in the intensity of gust fronts and subsequent updrafts (e.g., $[6,15,16])$. Hence, the differences in updraft mass fluxes in Figure 5(b) despite the fixed CDNC and the removed ice processes may have been caused by changes in rain evaporation, which are induced by aerosol, and those in the gust front intensity. Motivated by this, the control-BINnoice-cdnc run, the $10 \mathrm{M}-\mathrm{BIN}$-noice-cdnc run, the controlBULK-noice-cdnc run, and the 10M-BULK-noice-cdnc run, as depicted in Figure 5(b), are repeated by additionally turning off rain evaporative cooling. In these sensitivity runs, rain evaporation affects the rain mass but does not affect temperature. These sensitivity simulations are the controlBIN-no-rain-evp run, the 10M-BIN-no-rain-evp run, the control-BULK-no-rain-evp run, and the 10M-BULK-norain-evp run. By turning off rain evaporative cooling, we remove a pathway for the aerosol to influence gust fronts through aerosol effects on rain evaporative cooling. The flux profile shapes are now more consistent amongst simulations with the rain evaporative cooling turned off (Figure $7(\mathrm{a})$ ). The altitude of the updraft-mass-flux peak is $3.6-3.8 \mathrm{~km}$ among the "no-rain-evp" runs, which is similar to $3.9 \mathrm{~km}$ in the control-BIN run and the $10 \mathrm{M}-\mathrm{BIN}$ run. The controlBIN-no-rain-evp run and the 10M-BIN-no-rain-evp run in Figure 7(a) still show strong aerosol influences on the updraft mass fluxes in comparison with those in the controlBIN-noice-cdnc run and the 10M-BIN-noice-cdnc run in Figure 5(b). However, aerosol-related differences are small in the control-BULK-no-rain-evp run and the 10M-BULKno-rain-evp run in Figure 7(a) in comparison with those in the control-BULK-noice-cdnc run and the 10M-BULKnoice-cdnc run in Figure 5(b). Associated with this, the peak value of the updraft-mass-flux profile varies slightly by $2 \%$ from $0.41 \mathrm{~kg} \cdot \mathrm{m}^{-2} \cdot \mathrm{s}^{-1}$ in the control-BULK-no-rain-evp run to $0.42 \mathrm{~kg} \cdot \mathrm{m}^{-2} \cdot \mathrm{s}^{-1}$ in the $10 \mathrm{M}-\mathrm{BULK}-\mathrm{no}-$ rain-evp run, while the value varies by $9 \%$ from $0.44 \mathrm{~kg} \cdot \mathrm{m}^{-2} \cdot \mathrm{s}^{-1}$ in the control-BIN-no-rain-evp run to $0.48 \mathrm{~kg} \cdot \mathrm{m}^{-2} \cdot \mathrm{s}^{-1}$ in the $10 \mathrm{M}-$ BIN-no-rain-evp run. Comparing the simulations in Figures 5(b) to those in Figure 7(a), it appears that aerosol responses in BULK are controlled to a large extent by rain evaporative cooling and gust front generation; however, those responses in BIN appear to be less sensitive to aerosol influences on rain evaporation.

4.8. Cooling from Cloud-Liquid Evaporation. Recent studies have shown that not only rain evaporative cooling but also cloud-liquid evaporative cooling can affect the intensity of gust fronts and aerosol effects on it. Hence, we hypothesize that different treatments in the cloud-liquid evaporation may explain some of the remaining differences in Figure 7(a). Based on this hypothesis, we repeat the controlBIN-no-rain-evp run, the 10M-BIN-no-rain-evp run, the control-BULK-no-rain-evp run, and the 10M-BULK-norain-evp run in Figure 7 (a) by additionally turning off cloud-liquid evaporative cooling. In these sensitivity runs, which are the control-BIN-no-cld-evp run, the 10M-BINno-cld-evp run, the control-BULK-no-cld-evp run, and the 10M-BULK-no-cld-evp run, cloud-liquid evaporation affects the cloud-liquid mass, but temperature is not altered by cloud-liquid evaporation. The control-BIN-no-cld-evp run and the 10M-BIN-no-cld-evp run in Figure 7(b) show that updraft mass fluxes are now much smaller and exhibit little aerosol influences as compared to the control-BIN-no-rain- 


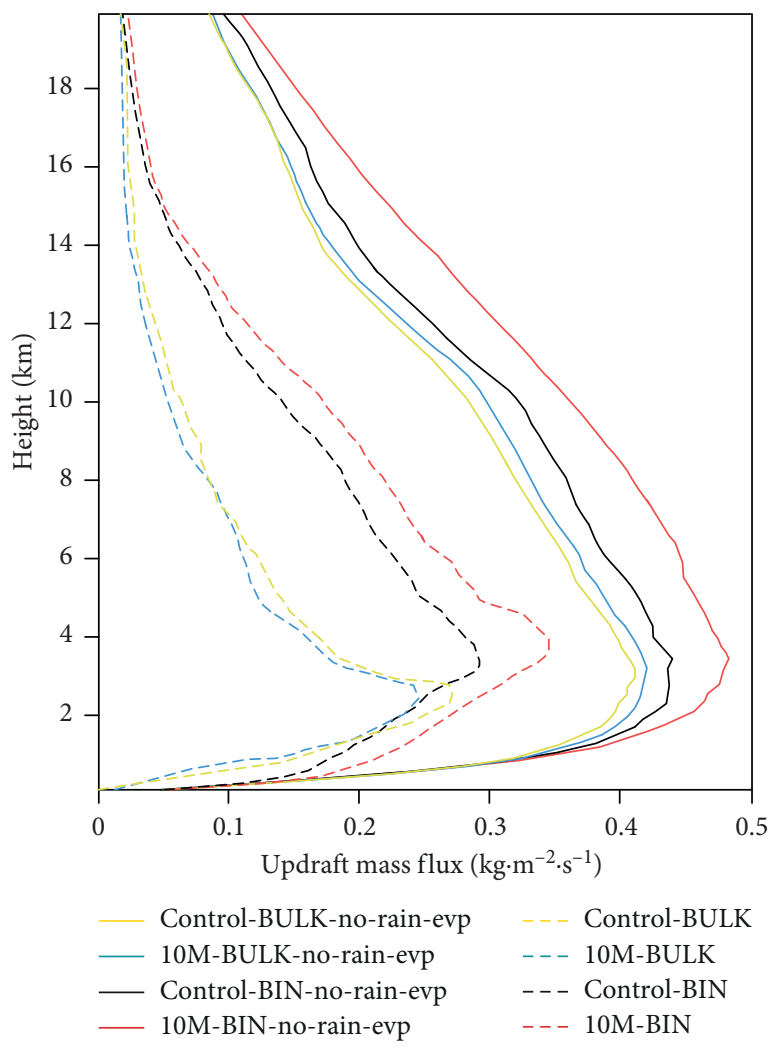

(a)

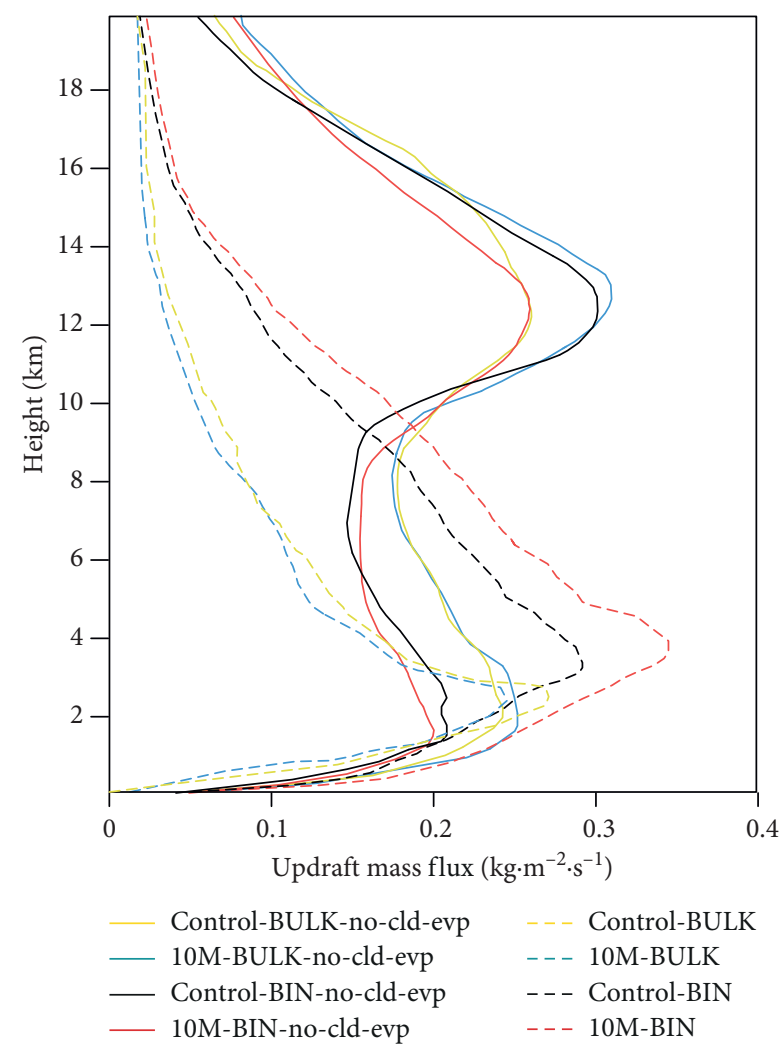

(b)

FIgURE 7: (a) Vertical distributions of the time- and domain-averaged updraft mass fluxes for the standard runs, the control-BIN-no-rainevp run, the 10M-BIN-no-rain-evp run, the control-BULK-no-rain-evp run, and the 10M-BULK-no-rain-evp run. (b) Vertical distributions of the time- and domain-averaged updraft mass fluxes for the standard runs, the control-BIN-no-cld-evp run, the 10M-BIN-no-cld-evp run, the control-BULK-no-cld-evp run, and the 10M-BULK-no-cld-evp run.

evp run and the 10M-BIN-no-rain-evp run in Figure 7(a), particularly at altitudes below the tropopause where clouds form. The tropopause is located around $12 \mathrm{~km}$. Associated with this, below the tropopause, the peak value of the updraftmass-flux profile varies slightly by $2 \%$ from $0.20 \mathrm{~kg} \cdot \mathrm{m}^{-2} \cdot \mathrm{s}^{-1}$ in the $10 \mathrm{M}-\mathrm{BIN}$-no-cld-evp run to $0.21 \mathrm{~kg} \cdot \mathrm{m}^{-2} \cdot \mathrm{s}^{-1}$ in the control-BIN-no-cld-evp run. The altitude of the peak is $1.6 \mathrm{~km}$, which is identical between the 10M-BIN-no-cld-evp run and the control-BIN-no-cld-evp run. The peak value is $52 \%$ smaller in the control-BIN-no-cld-evp run as compared to the control-BIN-no-rain-evp run, and it is $58 \%$ smaller in the 10M-BIN-no-cld-evp run as compared to the 10M-BINno-rain-evp run. The mass fluxes in the control-BULK-nocld-evp run and the 10M-BULK-no-cld-evp run in Figure 7 (b) also decrease in magnitude as compared to the control-BULK-no-rain-evp run and the 10M-BULK-no-rainevp run in Figure 7(a), although to a lesser extent, particularly at altitudes below the tropopause. Aerosol-related differences are also small between the control-BULK-no-cld-evp run and the 10M-BULK-no-cld-evp run below the tropopause (Figure 7(b)). Related to this, below the tropopause, the peak value varies slightly by $2 \%$ from $0.24 \mathrm{~kg} \cdot \mathrm{m}^{-2} \cdot \mathrm{s}^{-1}$ in the controlBULK-no-cld-evp run to $0.25 \mathrm{~kg} \cdot \mathrm{m}^{-2} \cdot \mathrm{s}^{-1}$ in the $10 \mathrm{M}-\mathrm{BULK}-$ no-cld-evp run. The altitude of the peak is $1.7-1.8 \mathrm{~km}$, which is very similar between the control-BULK-no-cld-evp run and the 10M-BULK-no-cld-evp run. The peak value is $41 \%$ smaller in the control-BULK-no-cld-evp run as compared to the control-BULK-no-rain-evp run, and it is $40 \%$ smaller in the 10M-BULK-no-cld-evp run as compared to the 10MBULK-no-rain-evp run.

While mass flux profile shapes are similar between the sensitivity simulations, as seen in Figure $7(\mathrm{~b})$, there are still significant differences in the magnitudes between the pair of the control-BIN-no-cld-evp run and the 10M-BIN-no-cldevp run and that of the control-BULK-no-cld-evp run and the 10M-BULK-no-cld-evp run below the tropopause (order $30 \%)$. Overall, it can be said that both rain evaporative cooling and cloud-liquid evaporative cooling have strong leverage on the mass flux profiles and the aerosol influence thereon.

4.9. Saturation. We hypothesize that the treatment of saturation might explain remaining differences in Figure 7(b). To check this hypothesis, the control-BIN-no-cld-evp run and the 10M-BIN-no-cld-evp run in Figure 7(b) are repeated using the saturation adjustment treatment in BULK. These sensitivity simulations are the control-BIN-sat run and the $10 \mathrm{M}-\mathrm{BIN}$-sat run, and results from these sensitivity simulations are presented in Figure 8. Differences in the magnitude of the updraft mass fluxes between the pair of the control-BIN-sat run and the 10M-BIN-sat run and that of 


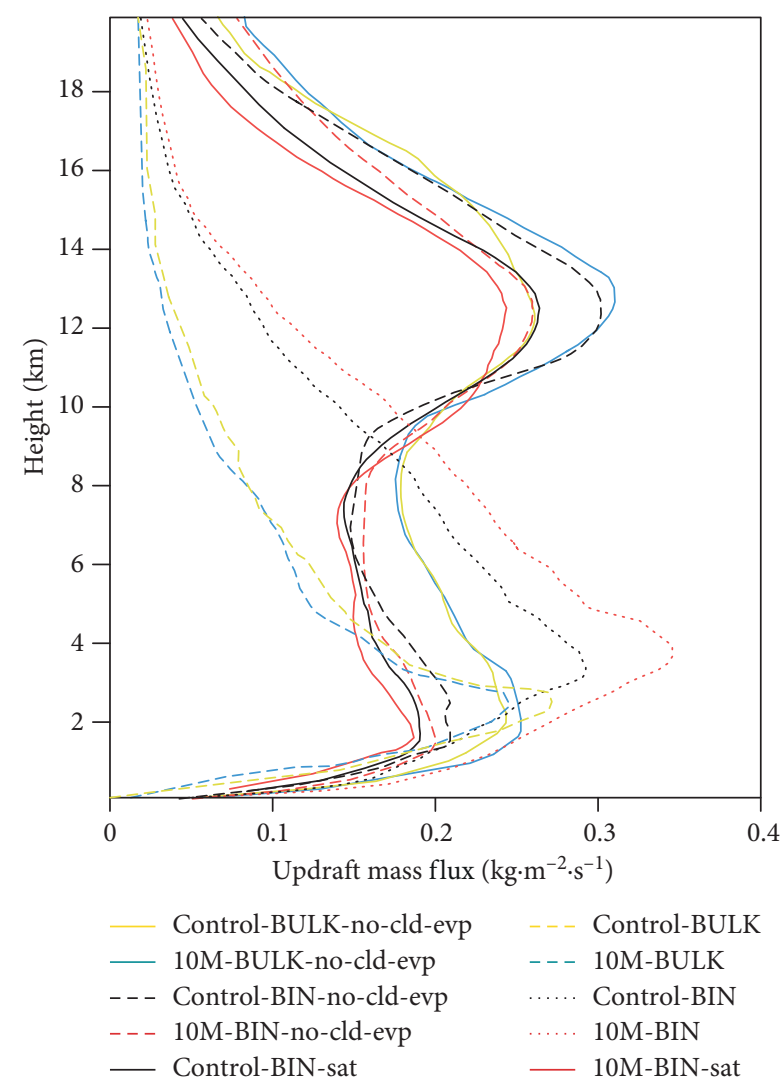

FIgURE 8: Vertical distributions of the time- and domain-averaged updraft mass fluxes for the simulations in Figure 7(b), the control$\mathrm{BIN}$-sat run, and the 10M-BIN-sat run.

the control-BULK-no-cld-evp run and the 10M-BULK-nocld-evp run in Figure 8 are now slightly larger than those between the pair of the control-BIN-no-cld-evp run and the 10M-BIN-no-cld-evp run and the pair of the control-BULKno-cld-evp run and the 10M-BULK-no-cld-evp run below the tropopause; note that the control-BIN-no-cld-evp run and the 10M-BIN-no-cld-evp run in Figure 7(b) are shown with dashed lines in Figure 8 and in Figure 8, and the control-BULK-no-cld-evp run and the con-BULK-no-cldevp run in Figure $7(\mathrm{~b})$ are shown with the same lines as in Figure 7(b). Regarding the differences which get larger, the peak value of the updrafts mass fluxes reduces from $0.21 \mathrm{~kg} \cdot \mathrm{m}^{-2} \cdot \mathrm{s}^{-1}$ in the control-BIN-no-cld-evp run to $0.19 \mathrm{~kg} \cdot \mathrm{m}^{-2} \cdot \mathrm{s}^{-1}$ in the control-BIN-sat run, while it reduces from $0.20 \mathrm{~kg} \cdot \mathrm{m}^{-2} \cdot \mathrm{s}^{-1}$ in the $10 \mathrm{M}-\mathrm{BIN}$-no-cld evp run to $0.18 \mathrm{~kg} \cdot \mathrm{m}^{-2} \cdot \mathrm{s}^{-1}$ in the $10 \mathrm{M}-\mathrm{BIN}$-sat run although the altitude of the peak value does not change among these runs. Here, we see that the different treatment of saturation does not help figure out the cause of the discrepancy between the BIN simulations and the BULK simulations, as shown in Figure 7(b).

4.10. Autoconversion and Accretion. We hypothesize that differences in the treatment of autoconversion and accretion of cloud ice and cloud liquid by precipitable hydrometeors may play a role in the differences in the updraft mass fluxes among the simulations in Figure 7(b). Based on this hypothesis, we repeat the simulations with BIN (i.e., the control-BIN-no-cld-evp run and the 10M-BIN-no-cld-evp run) in Figure 7 (b) by using the same autoconversion and accretion schemes as in the simulations with BULK (i.e., the control-BULK-no-cld-evp run and the 10M-BULK-no-cldevp run). These sensitivity simulations are the control-BINcol run and the 10M-BIN-col run. With the identical autoconversion and accretion schemes between the pair of the control-BIN-col run and the 10M-BIN-col run and the pair of the control-BULK-no-cld-evp run and the 10MBULK-no-cld-evp run, as seen in Figure 9, differences in the updraft-mass-flux profiles between these two pairs of simulations are now smaller as compared to the differences between the pair of the control-BULK-no-cld-evp run and the 10M-BULK-no-cld-evp run and that of the control-BINno-cld-evp run and the 10M-BIN-no-cld-evp run below the tropopause; note that the control-BIN-no-cld-evp run and the 10M-BIN-no-cld-evp run in Figure 7(b) are shown with dashed lines in Figure 9 and in Figure 9, and the controlBULK-no-cld-evp run and the con-BULK-no-cld-evp run in Figure 7(b) are shown with the same lines as in Figure 7(b). Regarding the differences which get smaller, the peak value of the updrafts mass fluxes increases by $10 \%$ from $0.21 \mathrm{~kg} \cdot \mathrm{m}^{-2} \cdot \mathrm{s}^{-1}$ in the control-BIN-no-cld-evp run to $0.23 \mathrm{~kg} \cdot \mathrm{m}^{-2} \cdot \mathrm{s}^{-1}$ in the control-BIN-col run, while it increases by $10 \%$ from $0.20 \mathrm{~kg} \cdot \mathrm{m}^{-2} \cdot \mathrm{s}^{-1}$ in the $10 \mathrm{M}$-BIN-no-cld evp run to $0.22 \mathrm{~kg} \cdot \mathrm{m}^{-2} \cdot \mathrm{s}^{-1}$ in the $10 \mathrm{M}-\mathrm{BIN}-\mathrm{col}$ run although the altitude of the peak value does not change among these runs. This results in around 10\% differences between the pair of the control-BIN-col run and the 10M-BIN-col run and the pair of the control-BULK-no-cld-evp run and the 10MBULK-no-cld-evp run, which are smaller than around $30 \%$ differences between the pair of the control-BIN-no-cldevp run and the 10M-BIN-no-cld-evp run and the pair of the control-BULK-no-cld-evp run and the 10M-BULK-no-cldevp run. However, in Figure 9, there are still remaining differences in the fluxes among the simulations. Both autoconversion and accretion treatments seem to be of importance but are insufficient in explaining all differences in Figure 7(b).

4.11. Sedimentation. We again hypothesize that one important remaining process-namely, sedimentation-might explain the remaining differences in Figure 7(b). Based on this hypothesis, the control-BIN-col run and the 10M-BINcol run in Figure 9 are repeated but with the version of sedimentation in BULK. These sensitivity simulations are the control-BIN-sed run and the 10M-BIN-sed run. As shown in Figure 10, differences, which are around 1-3\%, between the pair of the control-BIN-sed run and the 10MBIN-sed run and the pair of the control-BULK-no-cld-evp run and the 10M-BULK-no-cld-evp run are now very small as compared to differences, which are around $30 \%$, between the pair of the control-BIN-no-cld-evp run and the 10MBIN-no-cld-evp run and the pair of the control-BULK-nocld-evp run and the 10M-BULK-no-cld-evp run below the tropopause in Figure 7(b); note that the control-BIN-no-cld- 


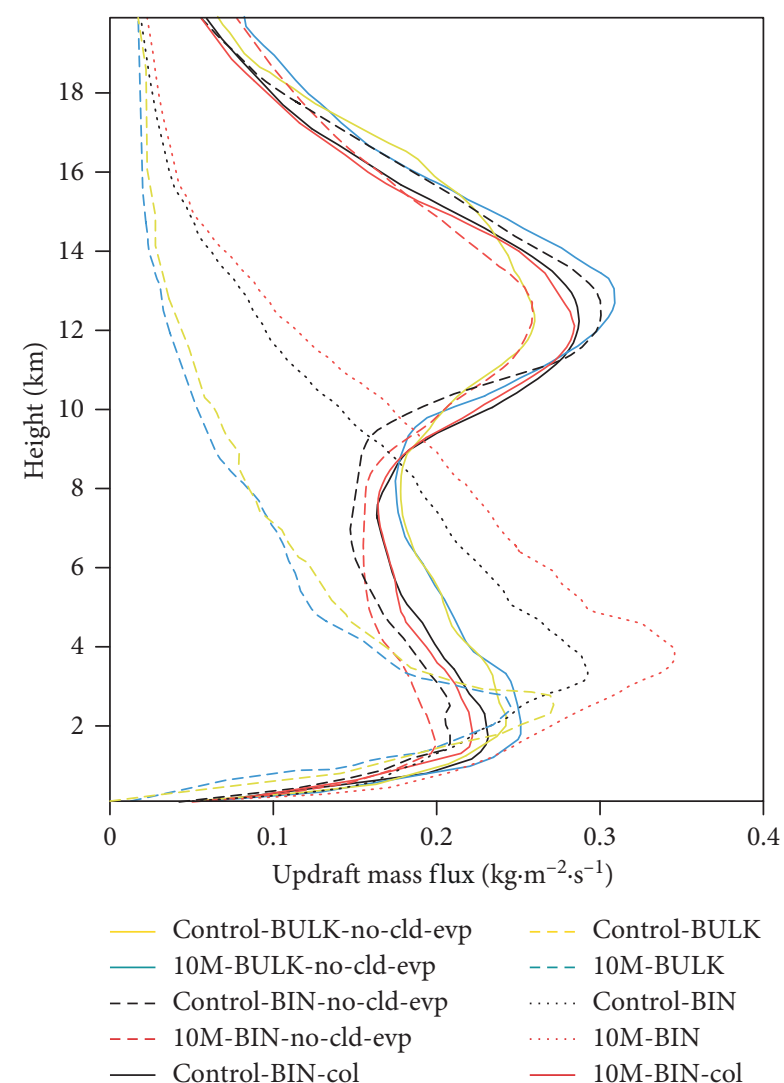

FIGURE 9: Vertical distributions of the time- and domain-averaged updraft mass fluxes for the simulations in Figure 7(b), the controlBIN-col run, and the 10M-BIN-col run.

evp run and the 10M-BIN-no-cld-evp run in Figure 7(b) are shown with dashed lines in Figure 10 and in Figure 10, and the control-BULK-no-cld-evp run and the con-BULK-nocld-evp run in Figure 7(b) are shown with the same lines as in Figure 7(b). Regarding this, the peak value in the updraft mass fluxes increases by $9 \%$ from $0.23 \mathrm{~kg} \cdot \mathrm{m}^{-2} \cdot \mathrm{s}^{-1}$ in the control-BIN-col run to $0.25 \mathrm{~kg} \cdot \mathrm{m}^{-2} \cdot \mathrm{s}^{-1}$ in the control-BINsed run, while it increases by $9 \%$ from $0.22 \mathrm{~kg} \cdot \mathrm{m}^{-2} \cdot \mathrm{s}^{-1}$ in the $10 \mathrm{M}-\mathrm{BIN}$-col run to $0.24 \mathrm{~kg} \cdot \mathrm{m}^{-2} \cdot \mathrm{s}^{-1}$ in the $10 \mathrm{M}-\mathrm{BIN}$-sed run. Sedimentation treatment appears to be of significant importance in making differences in updraft mass fluxes between the schemes.

4.12. Tests for Individual Effects of CDNC, Rain, and CloudLiquid Evaporative Cooling, Saturation, Collection, and Sedimentation Processes. Some of the simulations above involve multiple microphysical processes which are modified together. Hence, as described in Section 3.3, this prevents the isolation of individual effects of some of processes. To isolate effects of each of those processes, the standard simulations are repeated by modifying a process of interest only. The basic setup and naming of those repeated simulations are described in Section 3.3, and here, their results are described as follows.

As seen in the control-BIN-cdnc-only run, the 10MBIN-cdnc-only run, the control-BULK-cdnc-only run, and

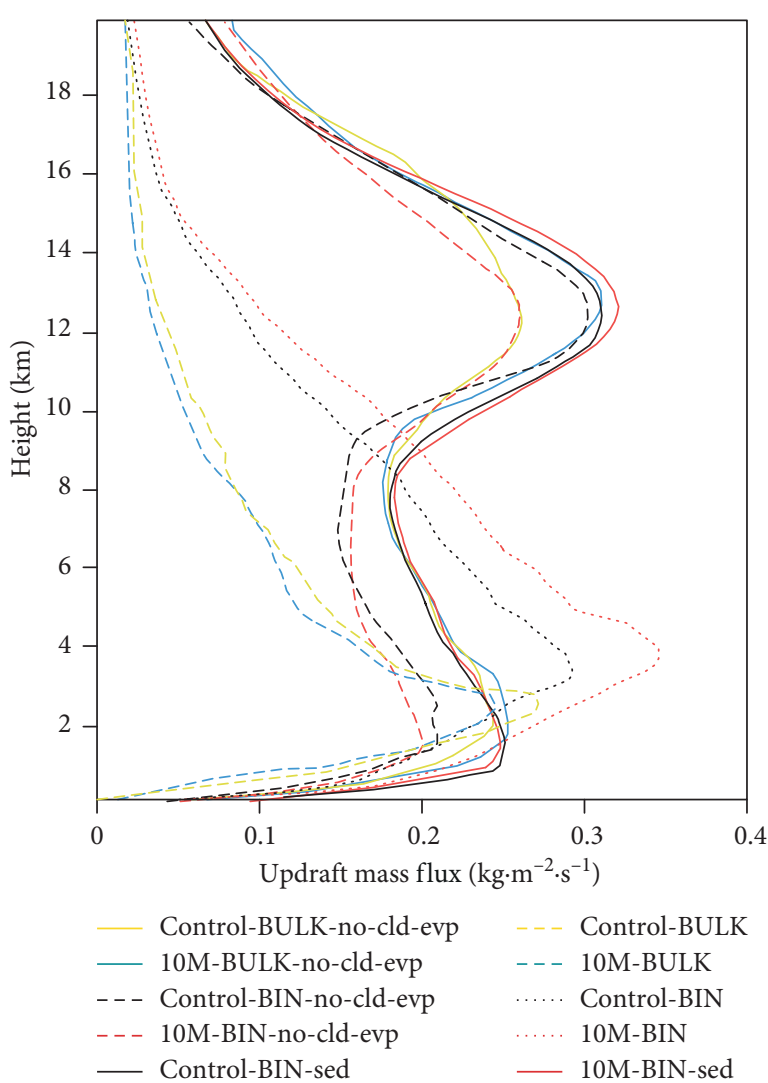

Figure 10: Vertical distributions of the time- and domain-averaged updraft mass fluxes for the simulations in Figure 7(b), the control$\mathrm{BIN}$-sed run, and the 10M-BIN-sed run.

the 10M-BULK-cdnc-only run in Figure 11(a), both BIN and BULK show aerosol-induced enhancement of updraft mass fluxes as shown in "noice-cdnc runs." Associated with this, the peak value of the updraft-mass-flux profile increases by $16 \%$ from $0.32 \mathrm{~kg} \cdot \mathrm{m}^{-2} \cdot \mathrm{s}^{-1}$ in the control-BIN-cdnc-only run to $0.37 \mathrm{~kg} \cdot \mathrm{m}^{-2} \cdot \mathrm{s}^{-1}$ in the $10 \mathrm{M}-\mathrm{BIN}$-cdnc-only run, while it increases by $11 \%$ from $0.27 \mathrm{~kg} \cdot \mathrm{m}^{-2} \cdot \mathrm{s}^{-1}$ in the control-BULKcdnc-only run to $0.30 \mathrm{~kg} \cdot \mathrm{m}^{-2} \cdot \mathrm{s}^{-1}$ in the $10 \mathrm{M}-\mathrm{BULK}-\mathrm{cdnc}-$ only run. The altitude of the peak is $3.9(2.8) \mathrm{km}$ in the control-BIN-cdnc-only run and the 10M-BIN-cdnc-only run (the control-BULK-cdnc-only run and the $10 \mathrm{M}$ BULK-cdnc-only run) as in the control-BIN run and the $10 \mathrm{M}-\mathrm{BIN}$ run (the control-BULK run and the 10M-BULK run). This confirms that different distributions of CDNC are the main cause of different responses of updraft mass fluxes to increasing aerosol concentration between the microphysical schemes, in comparison with the standard runs, as shown in "noice-cdnc" runs.

As seen in the control-BIN-no-rain-evp-only run, the 10M-BIN-no-rain-evp-only run, the control-BULK-norain-evp-only run, and the $10 \mathrm{M}$-no-rain-evp-only run in Figure 11(b), aerosol-induced differences are smaller between the control-BULK-no-rain-evp-only run and the 10M-BULK-no-rain-evp-only run as compared to a situation between the control-BULK run and 10M-BULK run. Associated with this, aerosol-induced difference in the peak value between the control-BULK-no-rain-evp-only run and 

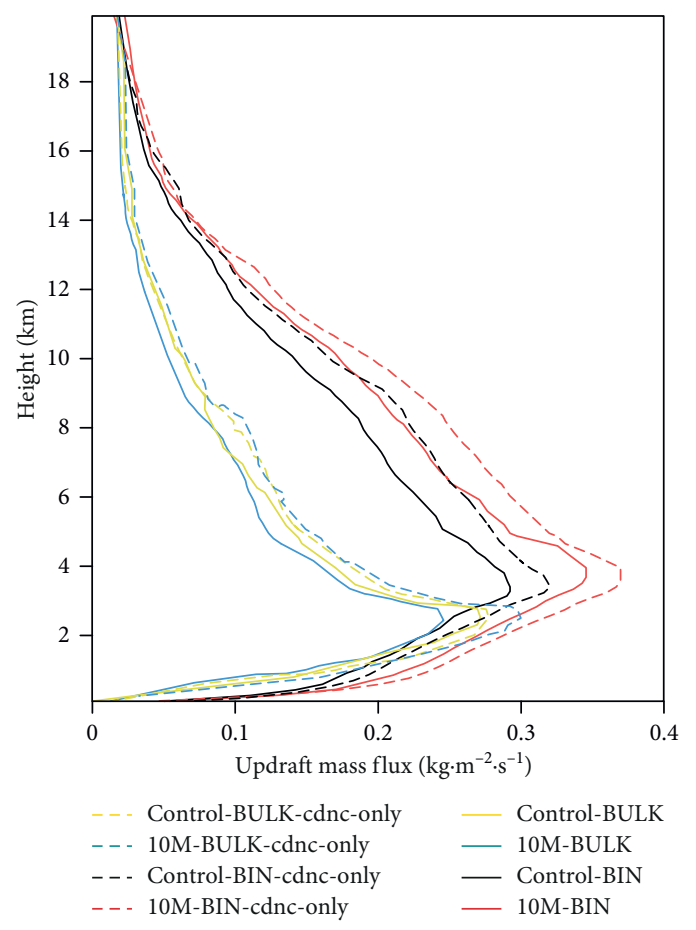

(a)

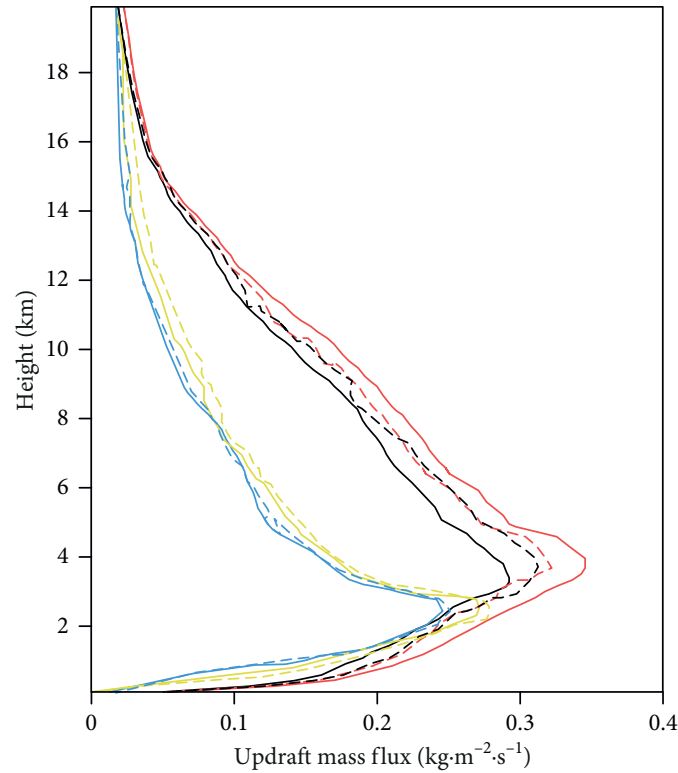

Control-BULK-no-cld-

- . - 10M-BULK-no-cld-evp-only — Control-BIN

- - - Control-BIN-no-cld-evp-only — 10M-BIN

- - - 10M-BIN-no-cld-evp-only

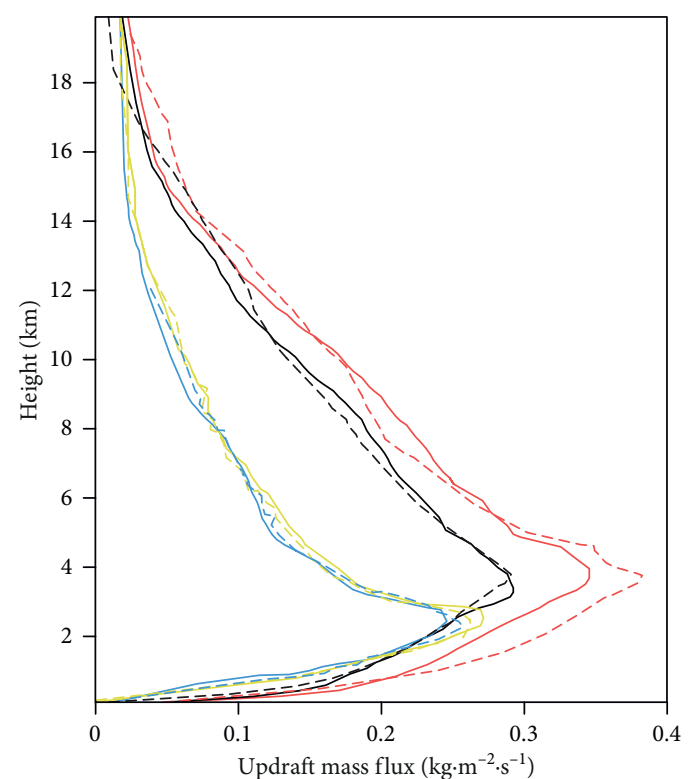

Control-BULK-no-rainevp-only

Control-BULK

- - 10M-BULK-no-rain-evp-only — Control-BIN

- - - Control-BIN-no-rain-evp-only _ 10M-BIN

- - - 10M-BIN-no-rain-evp-only

(b)

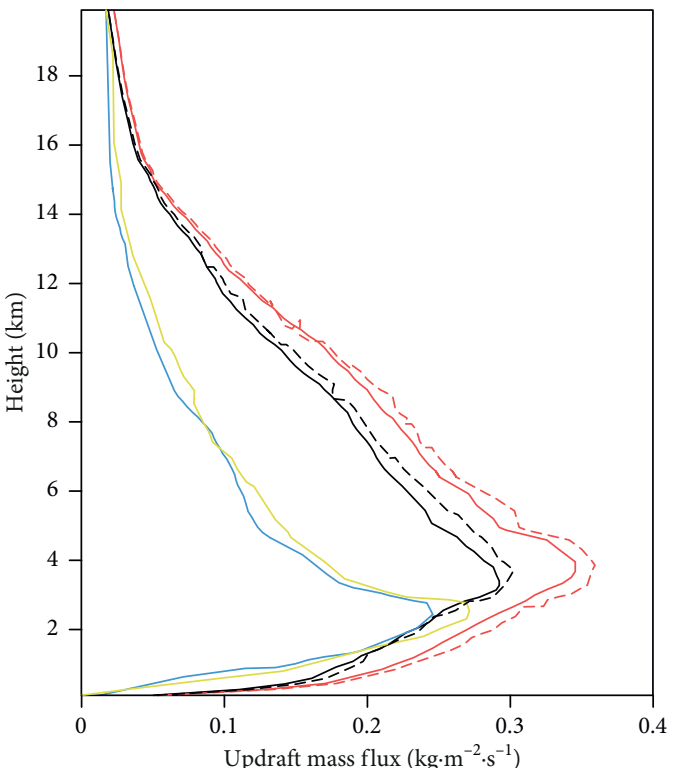

Control-BULK

10M-BULK

- - - Control-BIN-sat-only

- - 10M-BIN-sat-only

10M-BIN

(c)

Figure 11: Continued. 


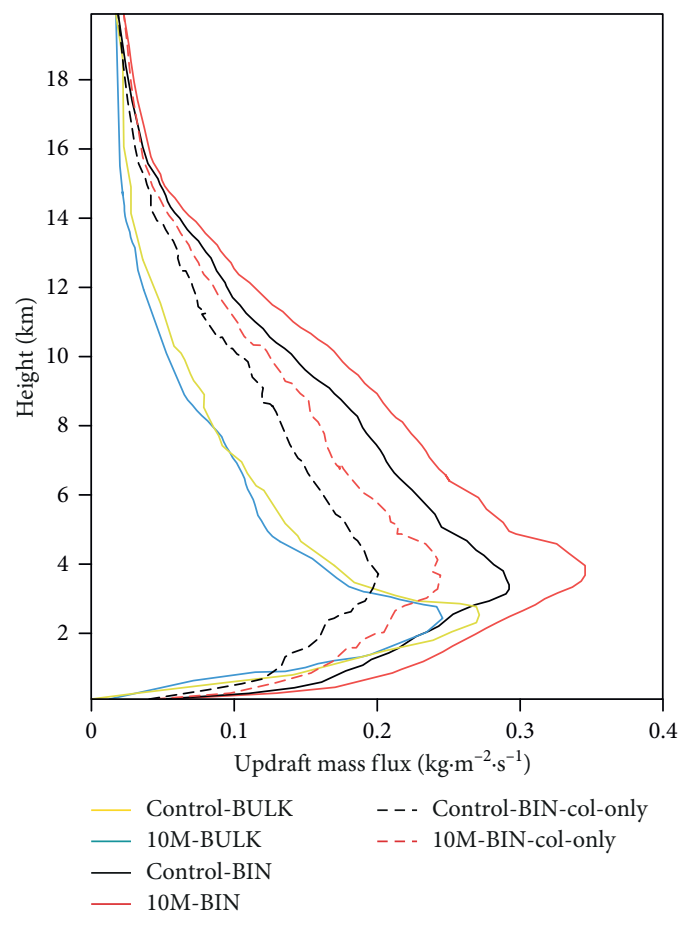

(e)

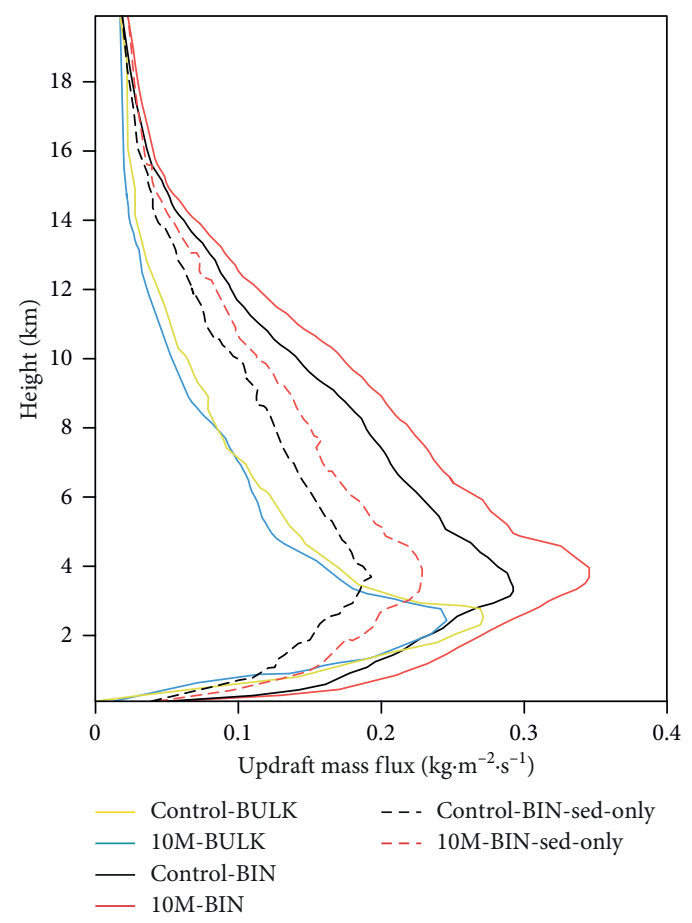

(f)

Figure 11: (a) Vertical distributions of the time- and domain-averaged updraft mass fluxes for the standard runs, the control-BIN-cdnconly run, the 10M-BIN-cdnc-only run, the control-BULK-cdnc-only run, and the 10M-BULK-cdnc-only run. (b) Vertical distributions of the time- and domain-averaged updraft mass fluxes for the standard runs, the control-BIN-no-rain-evp-only run, the 10M-BIN-no-rainevp-only run, the control-BULK-no-rain-evp-only run, and the 10M-BULK-no-rain-evp-only run. (c) Vertical distributions of the timeand domain-averaged updraft mass fluxes for the standard runs, the control-BIN-no-cld-evp-only run, the 10M-BIN-no-cld-evp-only run, the control-BULK-no-cld-evp-only run, and the 10M-BULK-no-cld-evp-only run. (d) Vertical distributions of the time- and domainaveraged updraft mass fluxes for the standard runs, the control-BIN-sat-only run, and the 10M-BIN-sat-only run. (e) Vertical distributions of the time- and domain-averaged updraft mass fluxes for the standard runs, the control-BIN-col-only run, and the 10M-BIN-col-only run. (f) Vertical distributions of the time- and domain-averaged updraft mass fluxes for the standard runs, the control-BIN-sed-only run, and the $10 \mathrm{M}$-BIN-sed-only run.

the 10M-BULK-no-rain-evp-only run is less than $0.01 \mathrm{~kg} \cdot \mathrm{m}^{-2} \cdot \mathrm{s}^{-1}$, and this is $80 \%$ smaller than that difference between the control-BULK run and the 10M-BULK run. The altitude of the peak in the control-BULK-no-rain-evp-only run and the 10M-BULK-no-rain-evp-only run is $2.8 \mathrm{~km}$ as in the control-BULK run and the $10 \mathrm{M}-\mathrm{BULK}$ run. However, aerosol-induced differences are greater between the controlBIN-no-rain-evp-only run and the 10M-BIN-no-rain-evponly run than between the control-BIN run and the 10MBIN run. Associated with this, the aerosol-induced difference in the peak value between the control-BIN-no-rainevp-only run and the $10 \mathrm{M}-\mathrm{BIN}$-no-rain-evp-only run is $0.09 \mathrm{~kg} \cdot \mathrm{m}^{-2} \cdot \mathrm{s}^{-1}$, and this is $29 \%$ greater than that difference between the control-BIN run and the 10M-BIN run. The altitude of the peak in the control-BIN-no-rain-evp-only run and the 10M-BIN-no-rain-evp-only run is $3.9 \mathrm{~km}$ as in the control-BIN run and the 10M-BIN run. This confirms that aerosol responses in BULK, but not BIN, are controlled to a large extent by rain evaporative cooling and gust front generation, as shown in "no-rain-evp" runs.

As seen in the control-BIN-no-cld-evp-only run, the 10M-BIN-no-cld-evp-only run, the control-BULK-no-cldevp-only run, and the 10M-BULK-no-cld-evp-only run in Figure 11(c), aerosol-induced differences are much smaller between the control-BIN-no-cld-evp-only run and the 10MBIN-no-cld-evp-only run as compared to a situation between the control-BIN run and 10M-BIN run. Associated with this, the aerosol-induced difference in the peak value between the control-BIN-no-cld-evp-only run and the $10 \mathrm{M}$ BIN-no-cld-evp-only run is $0.01 \mathrm{~kg} \cdot \mathrm{m}^{-2} \cdot \mathrm{s}^{-1}$, and this is $86 \%$ smaller than that difference between the control-BIN run and the 10M-BIN run. The altitude of the peak in the control-BIN-no-cld-evp-only run and the 10M-BIN-no-cldevp-only run is $3.9 \mathrm{~km}$ as in the control-BIN run and the $10 \mathrm{M}-\mathrm{BIN}$ run. However, the aerosol-induced difference between the control-BULK-no-cld-evp-only run and the $10 \mathrm{M}$-BULK-no-cld-evp-only run is $18 \%$ different from that between the control-BULK run and the 10M-BULK run in terms of the peak values. This demonstrates that aerosol responses in BIN, but not BULK, are controlled to a large extent by cloud-liquid evaporative cooling, as shown in "nocld-evp" runs.

As seen in Figure 11(d), differences between the pair of the control-BIN-sat-only run and the 10M-BIN-sat-only run and the pair of the control-BULK run and the 10M-BULK run are slightly greater than those between the pair of the control-BIN run and the 10M-BIN run and the pair of the control-BULK run and 10M-BULK run. Associated with 
this, a difference in the mean peak value between the pair of the control-BIN-sat-only run and the 10M-BIN-sat-only run and the pair of the control-BULK run and the 10M-BULK run is $33 \%$ greater than that between the pair of the controlBIN run and the 10M-BIN run and the pair of the controlBULK run and 10M-BULK run. The altitude of the peak in the control-BIN-sat-only run and the 10M-BIN-sat-only run is $3.9 \mathrm{~km}$ as in the control-BIN run and the $10 \mathrm{M}-\mathrm{BIN}$ run. Remember that the mean peak value between the controlBULK run and the $10 \mathrm{M}-\mathrm{BULK}$ run is $0.26 \mathrm{~kg} \cdot \mathrm{m}^{-2} \cdot \mathrm{s}^{-1}$, while the mean value between the control-BIN run and the $10 \mathrm{M}$ $\mathrm{BIN}$ run is $0.32 \mathrm{~kg} \cdot \mathrm{m}^{-2} \cdot \mathrm{s}^{-1}$. The mean peak value between the control-BIN-sat-only run and the $10 \mathrm{M}$-BIN-sat-only run is $0.34 \mathrm{~kg} \cdot \mathrm{m}^{-2} \cdot \mathrm{s}^{-1}$. This confirms that the different treatment of saturation is not able to explain the cause of the discrepancy between the BIN simulations and the BULK simulations. As seen in Figures 11(e) and 11(f), differences between the pair of the control-BIN-col-only run (the control-BIN-sed-only run) and the 10M-BIN-col-only run (the 10M-BIN-sed-only run) and the pair of the control-BULK run and 10M-BULK run are smaller than those between the pair of the control-BIN run and the 10M-BIN run and the pair of the control-BULK run and 10M-BULK run. Associated with this, a difference in the mean peak value between the pair of the control-BIN-colonly run (the control-BIN-sed-only run) and the 10M-BINcol-only run (the 10M-BIN-sed-only run) and the pair of the control-BULK run and 10M-BULK run is $50(33) \%$ smaller than that between the pair of the control-BIN run and the $10 \mathrm{M}-\mathrm{BIN}$ run and the pair of the control-BULK run and 10MBULK run. The mean peak value between the control-BINcol-only run (the control-BIN-sed-only run) and the $10 \mathrm{M}$ BIN-col-only run (the 10M-BIN-sed-only run) is $0.23(0.22)$ $\mathrm{kg} \cdot \mathrm{m}^{-2} \cdot \mathrm{s}^{-1}$. The altitude of the peak in the control-BIN-colonly run, the control-BIN-sed-only run, the $10 \mathrm{M}-\mathrm{BIN}-\mathrm{col}-$ only run, and the $10 \mathrm{M}$-BIN-sed-only run is $3.9 \mathrm{~km}$ as in the control-BIN run and the 10M-BIN run. This confirms that different autoconversion and accretion (sedimentation) treatment play an important role in explaining differences between the BIN simulations and the BULK simulations as shown in the control-BIN-col run and the 10M-BIN-col run (the control-BIN-sed run and the 10M-BIN-sed run).

\subsection{Two-Moment Prediction for Snow and Graupel. As} exemplified by Figure 12(a), comparisons among the control-BULK-2 mt run and the 10M-BULK-2 mt run with the two-moment prediction for snow and graupel and the previous runs with the one-moment prediction for snow and graupel as in the control-BULK run and the 10M-BULK run demonstrate that the qualitative nature of results does not vary with the varying prediction method for snow and graupel. However, as seen in Figure 12(a), with the use of the two-moment prediction, the altitude of the distribution peak and the peak value is lowered. The altitude of peak lowers by $32 \%$ from $2.8 \mathrm{~km}$ in the control-BULK run and the $10 \mathrm{M}$ BULK run to $1.9 \mathrm{~km}$ in the control-BULK- $2 \mathrm{mt}$ run and the $10 \mathrm{M}-\mathrm{BULK}-2 \mathrm{mt}$ run, while the peak value lowers by $3 \%$ from $0.27 \mathrm{~kg} \cdot \mathrm{m}^{-2} \cdot \mathrm{s}^{-1}$ in the control-BULK run to $0.26 \mathrm{~kg} \cdot \mathrm{m}^{-2} \cdot \mathrm{s}^{-1}$ in the control-BULK-2 $\mathrm{mt}$ run, and it lowers by $4 \%$ from $0.25 \mathrm{~kg} \cdot \mathrm{m}^{-2} \cdot \mathrm{s}^{-1}$ in the $10 \mathrm{M}-\mathrm{BULK}$ run to $0.24 \mathrm{~kg} \cdot \mathrm{m}^{-2} \cdot \mathrm{s}^{-1}$ in the $10 \mathrm{M}-\mathrm{BULK}-2 \mathrm{mt}$ run. Also, there is an increase in updraft mass fluxes above $4 \mathrm{~km}$ in the controlBULK-2 mt run, as compared to the control-BULK run, and in the 10M-BULK- $2 \mathrm{mt}$ run, as compared to the $10 \mathrm{M}$-BULK run. This is because the use of the two-moment scheme induces increases in the mass or loading of snow and graupel at low altitudes around $4 \mathrm{~km}$ and decreases in the mass at high altitudes above $4 \mathrm{~km}$, which is similar to findings by Wacker and Seifert [41].

Figure 10 shows the final comparison between BIN and BULK. Hence, as another good example of how the twomoment prediction affects results here, Figures 10 and 12(b) are compared. In Figure 12(b), results from the repeated control-BULK-no-cld-evp run and the 10M-BULK-no-cldevp run with two-moment prediction for snow and graupel are shown together with some of simulations that are depicted in Figure 10 and are the control-BIN-sed run, the 10M-BINsed run, the control-BIN-no-cld-evp run, and the 10M-BINno-cld-evp run; these sensitivity runs with two-moment prediction are the control-BULK-no-cld-evp- $2 \mathrm{mt}$ run and the 10M-BULK-no-cld-evp- $2 \mathrm{mt}$ run. Comparisons between Figures 10 and 12(b) demonstrate that whether graupel and snow adopt the two-moment prediction in BULK does not affect the very small differences in updraft mass fluxes between the pair of the control-BIN-sed run and the 10M-BINsed run and the pair of "BULK-no-cld-evp" runs.

\section{Summary and Conclusions}

This study mainly focuses on and examines key microphysical processes that cause differences in the simulations of clouds and aerosol-cloud interactions between two microphysical schemes, i.e., BIN and BULK. For this examination, this study focuses on differences in updrafts, which are represented by updraft mass fluxes, and their response to increasing aerosol concentration between BIN and BULK.

Aerosol-induced invigoration of convection is simulated with BIN, but not simulated with BULK. Stated differently, there are aerosol-induced increases in updraft mass fluxes with BIN, while there are no aerosol-induced those increases with BULK. The profile or pattern of the vertical distribution of updraft mass fluxes with BULK is substantially different from that with BIN in terms of the altitude and magnitude of the updraft-mass-flux peak and the vertical variation of updraft mass fluxes around it. Results here indicate that whether the invigoration is present or not is strongly dependent on how CDNC is predicted. The different pattern of the updraft-mass-flux distribution between the schemes is due to much larger snow mass and associated loading around the freezing level in the simulations with BULK than those with BIN. The much greater loading of snow mass hinders the growth of updraft mass fluxes around the freezing level. This lowers the altitude and magnitude of the updraft-mass-flux peak and causes much larger vertical variation of updraft mass fluxes around the altitude where the peak occurs in the BULK simulations. It is notable that the latent heating or cooling associated with ice processes does not affect the vertical pattern of updraft mass fluxes in 


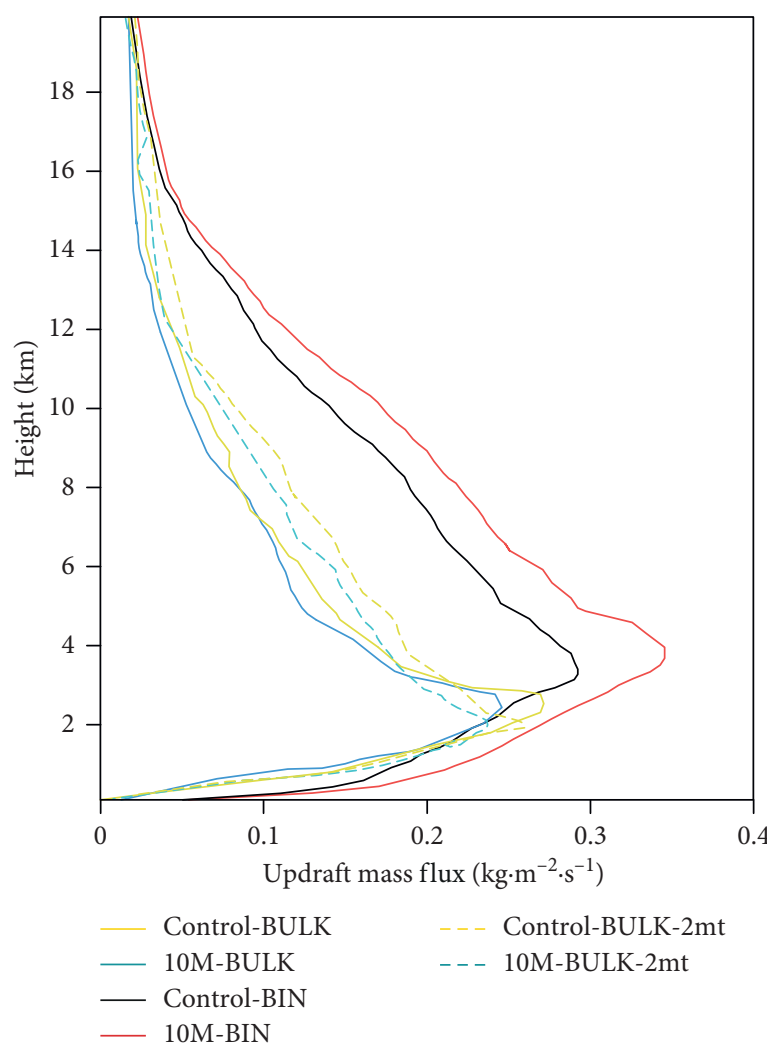

(a)

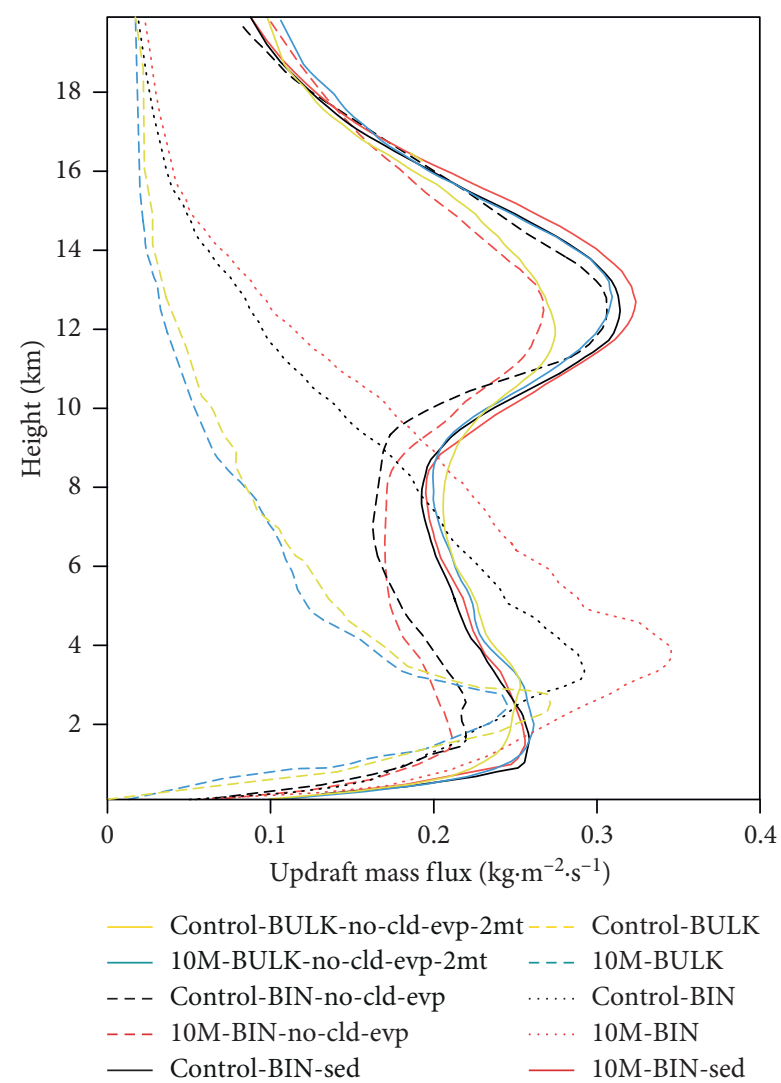

(b)

FIgURE 12: (a) Vertical distributions of the time- and domain-averaged updraft mass fluxes for the standard runs, the control-BULK-2 mt run, and the 10M-BULK-2 mt run. (b) Vertical distributions of the time- and domain-averaged updraft mass fluxes for the simulations in Figure 10 except for the fact that the control-BULK-no-cld-evp run and the 10M-BULK-no-cld-evp run are replaced with the controlBULK-no-cld-evp-2 $\mathrm{mt}$ run and the 10M-BULK-no-cld-evp-2 $\mathrm{mt}$ run.

the simulations with BULK much as compared to the loading of snow.

Although differences in the CDNC prediction and ice processes (including the snow process) are removed, thus, the differences in the invigoration are removed and those in the pattern of the vertical distribution of updraft mass fluxes are nearly removed between the simulations with BIN and those with BULK, there are still remaining differences. Among these remaining differences, differences between the high-aerosol case and the low-aerosol case for each of BIN and BULK are controlled by aerosol-induced changes in evaporation-related cooling and the associated gust front intensity. While the differences in updraft mass fluxes are explained by different rain evaporative cooling between the high-aerosol case and the low-aerosol case with BULK, those differences in cooling, due to rain evaporation, do not explain those differences in updraft mass fluxes with BIN. Those differences in updraft mass fluxes with BIN are explained by the differences in cloud-liquid evaporative cooling. This qualitative nature of roles by each of rain and cloud-liquid evaporative cooling in explaining differences between the high-aerosol case and the low-aerosol case for each of BIN and BULK is also produced in the repeated standard simulations where only rain or cloud-liquid evaporative cooling is turned off.
With the fixed CDNC, removed ice processes, and evaporative cooling, the vertical pattern of updraft mass fluxes shows negligible differences between simulations with BIN and those with BULK and the variation in updraft mass fluxes from the high-aerosol case to the low-aerosol case for each of BIN and BULK is nearly removed. However, there are still remaining differences in the magnitude of updraft mass fluxes between the simulations with BULK and those with BIN. These remaining differences are explained by different treatments of collection and sedimentation processes; however, the impact of the different treatments of saturation on the remaining differences is negligible. This qualitative nature of roles by collection, sedimentation, and saturation processes in explaining differences between the BULK simulations and the BIN simulations is also produced in the repeated standard runs where only each of these processes is tested.

There are around dozens or more of microphysical schemes that have been created. Also, each scheme has its own different versions. It would be the best strategy to compare all of those present schemes and their different versions for obtaining perfectly general conclusions. However, performing process-level research as shown in this paper by modifying each microphysical process in the model code requires a large amount of time and computer 
resources even for the comparison between the selected two schemes. Unfortunately, the funding, associated time, and computer resources given are not enough to perform process-level research for all of present microphysical schemes and their different versions. Maybe, comparing all of those present schemes for one or two specific processes such as autoconversion and/or sedimentation may be possible. There are studies such as Liu and Daum [44] and Lee and Baik [45] that compare more than two microphysical schemes for a specific process; however, these studies also do not deal with all of present schemes due to limit on time and computer resources. Considering the limit on time and resources, we have to make a compromise between processlevel research which tests numerous microphysical processes individually and the number of microphysical schemes that are tested. In the process of making the compromise, this study leans toward process-level research by sacrificing the number of microphysical schemes tested and associated generality of results. This is motivated by the fact that, as stated in introduction, the identification of key processes, which create the discrepancy in the simulation of the feedbacks between increasing aerosol concentration, microphysics, and dynamics among models, has been rarely performed, and the community does not have even a preliminary and basic understanding of those key processes themselves; note that the identification of key processes requires process-level research as shown in this study. Hence, through process-level research, this study provides preliminary, though not general, information on those key processes, by comparing the limited number of schemes, as a stepping stone to the general information which can be pursued in the future studies. Considering that the information on those key processes has been near absent, it is believed that this preliminary information itself is valuable by providing a preliminary clue to how to approach the discrepancy among models, despite the fact that comparisons for the limited number of schemes are not as perfect as those between all of the present schemes.

It should be reiterated that the main goal of this study is to identify "key" processes, but not all processes, which contribute to differences in updrafts and their responses to increasing aerosol concentration between the schemes. The first reason for not focusing on all those processes is that we even do not know what those key processes are to say nothing of all those processes and thus identifying those key processes itself can be an important key stepping stone to the understanding of how differences in updrafts and their responses are created between BIN and BULK. It should be noted that, as seen in Section 4.11 and Figure 10, sensitivity tests for BULK and BIN show similar results in the troposphere where clouds form, and this indicates that processes tested in this study are key and main processes that explain most of differences in updrafts and their responses between BULK and BIN. However, it is also true that, as shown in Figure 10, although most of differences between BULK and BIN are explained by those key processes, BULK and BIN do not show 100\% perfect consistency between them, and there is a small remaining difference between BULK and BIN. This small difference might be explained by processes not tested in this study. Despite this, it is believed that the fact that processes tested in this study explain most of differences between the schemes should be considered as an important finding based on which we can gain a preliminary understanding of key processes, and thus, this study achieves its main goal.

\section{Data Availability}

The data used are currently private and stored in our private computer system. Opening the data to the public requires approval from the funding source. Since the funding project associated with this work is still going on, the source does not allow the data to be open to the public, and 2-3 years after the project ends, the data can be open to the public. However, if there is any inquiry about the data, contact the corresponding author Seoung Soo Lee (cumulss@gmail.com).

\section{Disclosure}

Seoung Soo Lee is now at Department of Meteorology, San Jose State University, San Jose, CA, USA.

\section{Conflicts of Interest}

The authors declare that they have no conflicts of interest.

\section{Acknowledgments}

This study was supported by NOAA (NOAA-NWS-NWSPO2015-2004117), NASA/MUREP Cooperative Agreement (NNX15AQ02A), the Ministry of Education (NRF-2018R1D 1A1A09083227), and the National Strategic Project-Fine Particle of the National Research Foundation of Korea (NRF) funded by the Ministry of Science and ICT (MSIT), the Ministry of Environment (MOE), and the Ministry of Health and Welfare (MOHW) (NRF-2017M3D8A1092022). It was also supported by the National Institute of Environment Research (NIER), funded by the MOE (NIER-2018-01-02-033), and via Public Technology Program Based on Environmental Policy (2017000160003).

\section{References}

[1] M. Bollasina and S. Nigam, "Indian ocean SST, evaporation, and precipitation during the South Asian summer monsoon in IPCC-AR4 coupled simulations," Climate Dynamics, vol. 33, no. 7-8, pp. 1017-1032, 2009.

[2] S. S. Lee, B.-G. Kim, C. Lee, S. S. Yum, and D. Posselt, "Effect of aerosol pollution on clouds and its dependence on precipitation intensity," Climate Dynamics, vol. 42, no. 3-4, pp. 557-577, 2014.

[3] S. S. Lee, B.-G. Kim, S. S. Yum et al., "Effects of aerosol on evaporation, freezing and precipitation in a multiple cloud system," Climate Dynamics, vol. 48, no. 3-4, pp. 1069-1087, 2016.

[4] S. S. Lee, Z. Li, J. Mok et al., "Interactions between aerosol absorption, thermodynamics, dynamics, and microphysics and their impacts on a multiple-cloud system," Climate Dynamics, vol. 49, no. 11-12, pp. 3905-3921, 2017. 
[5] M. G. Manoj, P. C. S. Devara, P. D. Safai, and B. N. Goswami, "Absorbing aerosols facilitate transition of Indian monsoon breaks to active spells," Climate Dynamics, vol. 37, no. 11-12, pp. 2181-2198, 2010.

[6] R. L. Goswami, S. C. van den Heever, and G. L. Stephens, "Modeling aerosol impacts on convective storms in different environments," Journal of the Atmospheric Sciences, vol. 67, no. 12, pp. 3904-3915, 2010.

[7] Z. Wang, H. Zhang, and X. Zhang, "Projected response of East Asian summer monsoon system to future reductions in emissions of anthropogenic aerosols and their precursors," Climate Dynamics, vol. 47, no. 5-6, pp. 1455-1468, 2016.

[8] D. L. Hartmann, Global Physical Climatology, Academic Press, Cambridge, MA, USA, 1994.

[9] R. A. Houze, Cloud Dynamics, Academic Press, Cambridge, MA, USA, 1993.

[10] V. Ramanathan, R. D. Cess, E. F. Harrison et al., "Cloudradiative forcing and climate: results from the earth radiation budget experiment," Science, vol. 243, no. 4887, pp. 57-63, 1989.

[11] D. Rosenfeld, U. Lohmann, G. B. Raga et al., "Flood or drought: how do aerosols affect precipitation?," Science, vol. 321, no. 5894, pp. 1309-1313, 2008.

[12] W.-K. Tao, J.-P. Chen, Z. Li, C. Wang, and C. Zhang, "Impact of aerosols on convective clouds and precipitation," Reviews of Geophysics, vol. 50, no. 2, article RG2001, 2012.

[13] S. S. Lee, "Aerosols, clouds and climate," Nature Geoscience, vol. 4, no. 12, pp. 826-827, 2011.

[14] S. S. Lee, L. J. Donner, and J. E. Penner, “Thunderstorm and stratocumulus: how does their contrasting morphology affect their interactions with aerosols?," Atmospheric Chemistry and Physics, vol. 10, no. 14, pp. 6819-6837, 2010.

[15] W.-K. Tao, X. Li, A. Khain, T. Matsui, S. Lang, and J. Simpson, "Role of atmospheric aerosol concentration on deep convective precipitation: cloud-resolving model simulations," Journal of Geophysical Research, vol. 112, no. 24, article D24S18, 2007.

[16] S. C. van den Heever and W. R. Cotton, "Urban aerosol impacts on downwind convective storms," Journal of Applied Meteorology and Climatology, vol. 46, no. 6, pp. 828-850, 2007.

[17] S.-S. Lee and G. Feingold, "Aerosol effects on the cloud-field properties of tropical convective clouds," Atmospheric Chemistry and Physics, vol. 13, no. 14, pp. 6713-6726, 2013.

[18] J. Fan, T. Yuan, J. M. Comstock et al., "Dominant role by vertical wind shear in regulating aerosol effects on deep convective clouds," Journal of Geophysical Research, vol. 114, no. 22, article D22206, 2009.

[19] A. Khain, N. BenMoshe, and A. Pokrovsky, "Factors determining the impact of aerosols on surface precipitation from clouds: an attempt at classification," Journal of the Atmospheric Sciences, vol. 65, no. 6, pp. 721-1748, 2009.

[20] S. S. Lee, L. J. Donner, V. T. J. Phillips, and Y. Ming, "The dependence of aerosol effects on clouds and precipitation on cloud-system organization, shear and stability," Journal of Geophysical Research, vol. 113, no. 16, article D16202, 2008.

[21] A. P. Khain, A. Heymsfield, A. Korolev et al., "Representation of microphysical processes in cloud-resolving models: spectral (bin) microphysics versus bulk parameterization," Reviews of Geophysics, vol. 53, no. 2, pp. 247-322, 2015.

[22] W.-K. Tao, R. Atlas, D. Randall et al., "A multiscale modeling system: developments, applications, and critical issues," Bulletin of the American Meteorological Society, vol. 90, no. 4, pp. 515-534, 2009.
[23] W.-K. Tao, J. Chern, J. Entin et al., "The Goddard multi-scale modeling system with unified physics," Annales Geophysicae, vol. 27, no. 8, pp. 3055-3064, 2009.

[24] Y. Wang, J. Fan, R. Zhang, L. R. Leung, and C. Franklin, "Improving bulk microphysics parameterizations in simulations of aerosol effects," Journal of Geophysical Research: Atmospheres, vol. 118, no. 11, pp. 5361-5379, 2013.

[25] J. Fan, L. R. Leung, Z. Li et al., "Aerosol impacts on clouds and precipitation in Eastern China: results from bin and bulk microphysics," Journal of Geophysical Research: Atmospheres, vol. 117, no. 16, article D00K36, 2012.

[26] J. Fan, Y.-C. Liu, K.-M. Xu et al., "Improving representation of convective transport for scale-aware parameterization: 1. Convection and cloud properties simulated with spectral bin and bulk microphysics," Journal of Geophysical Research: Atmospheres, vol. 120, no. 8, pp. 3485-3509, 2015.

[27] T. Iguchi, T. Nakajima, A. P. Khain et al., "Evaluation of cloud microphysics in JMA-NHM simulations using bin or bulk microphysical schemes through comparison with cloud radar observations," Journal of the Atmospheric Sciences, vol. 69, no. 8, pp. 2566-2586, 2012.

[28] A. Khain and B. Lynn, "Simulation of a supercell storm in clean and dirty atmosphere using weather research and forecast model with spectral bin microphysics," Journal of Geophysical Research, vol. 114, no. 19, article D19209, 2009.

[29] G. Thompson and T. Eidhammer, "A study of aerosol impacts on clouds and precipitation development in a large winter cyclone," Journal of the Atmospheric Sciences, vol. 71, no. 10, pp. 3636-3658, 2014.

[30] D. T. Dawson, M. Xue, J. A. Milbrandt, and M. K. Yau, "Comparison of evaporation and cold pool development between single-moment and multimoment bulk microphysics schemes in idealized simulations of tornadic thunderstorms," Monthly Weather Review, vol. 138, no. 4, pp. 1152-1171, 2010.

[31] H. Morrison and J. Milbrandt, "Comparison of two-moment bulk microphysics schemes in idealized supercell thunderstorm simulations," Monthly Weather Review, vol. 139, no. 4, pp. 1103-1130, 2011.

[32] S. Solomon, D. Qin, M. Manning et al., "Technical summary," in Climate Change 2007: The Physical Science Basis. Contribution of Working Group I to the Fourth Assessment Report of the Intergovernmental Panel on Climate Change, S. Solomon, D. Qin, M. Manning et al., Eds., Cambridge University Press, Cambridge, UK, 2007.

[33] H. Wang, W. C. Skamarock, and G. Feingold, "Evaluation of scalar advection schemes in the advanced research WRF model using large-eddy simulations of aerosol-cloud interactions," Monthly Weather Review, vol. 137, no. 8, pp. 2547-2558, 2009.

[34] E. J. Mlawer, S. J. Taubman, P. D. Brown, M. J. Iacono, and S. A. Clough, "Radiative transfer for inhomogeneous atmospheres: RRTM, a validated correlated-k model for the longwave," Journal of Geophysical Research: Atmospheres, vol. 102, no. 14, pp. 16663-16682, 1997.

[35] Y. Fouquart and B. Bonnel, "Computations of solar heating of the earth's atmosphere: a new parameterization," Beitraege zur Physik der Atmosphaere, vol. 53, pp. 35-62, 1980.

[36] P. T. May, C. Jakob, J. H. Mather, and G. Vaughan, "Characterizing oceanic convective cloud systems-the tropical warm pool international cloud experiment," Bulletin of the American Meteorological Society, vol. 89, no. 2, pp. 153-155, 2008. 
[37] A. Fridlind, "ARM/GCSS/SPARC TWP-ICE CRM intercomparison study," 2010, http://pubs.giss.nasa.gov/abs/ fr08100v.html.

[38] R. R. Rogers and M. K. Yau, A Short Course in Cloud Physics, Pergamon Press, Oxford, UK, 1991.

[39] A. Khain, D. Rosenfeld, and A. Pokrovsky, "Aerosol impact on the dynamics and microphysics of deep convective clouds," Quarterly Journal of the Royal Meteorological Society, vol. 131, no. 611, pp. 2639-2663, 2005.

[40] S. C. van den Heever, G. G. Carrió, W. R. Cotton, P. J. DeMott, and A. J. Prenni, "Impacts of nucleating aerosol on Florida storms. Part I: mesoscale simulations," Journal of the Atmospheric Sciences, vol. 63, no. 7, pp. 1752-1775, 2006.

[41] U. Wacker and A. Seifert, "Evolution of rain water profiles resulting from pure sedimentation: spectral vs. parameterized description," Atmospheric Research, vol. 58, no. 1, pp. 19-39, 2001.

[42] J. A. Milbrandt and M. K. Yau, "A multimoment bulk microphysics parameterization. Part I: analysis of the role of the spectral shape parameter," Journal of the Atmospheric Sciences, vol. 62, no. 9, pp. 3051-3064, 2005.

[43] J. A. Milbrandt and R. McTaggart-Cowan, "Sedimentationinduced errors in bulk microphysics schemes," Journal of the Atmospheric Sciences, vol. 67, no. 12, pp. 3931-3948, 2010.

[44] Y. Liu and P. H. Daum, "Parameterization of the autoconversion process. Part I: analytical formulation of the kessler-type parameterizations," Journal of the Atmospheric Sciences, vol. 61, no. 13, pp. 1539-1548, 2004.

[45] H. Lee and J.-J. Baik, "A physically based autoconversion parameterization," Journal of the Atmospheric Sciences, vol. 74, no. 5, pp. 1599-1616, 2017. 

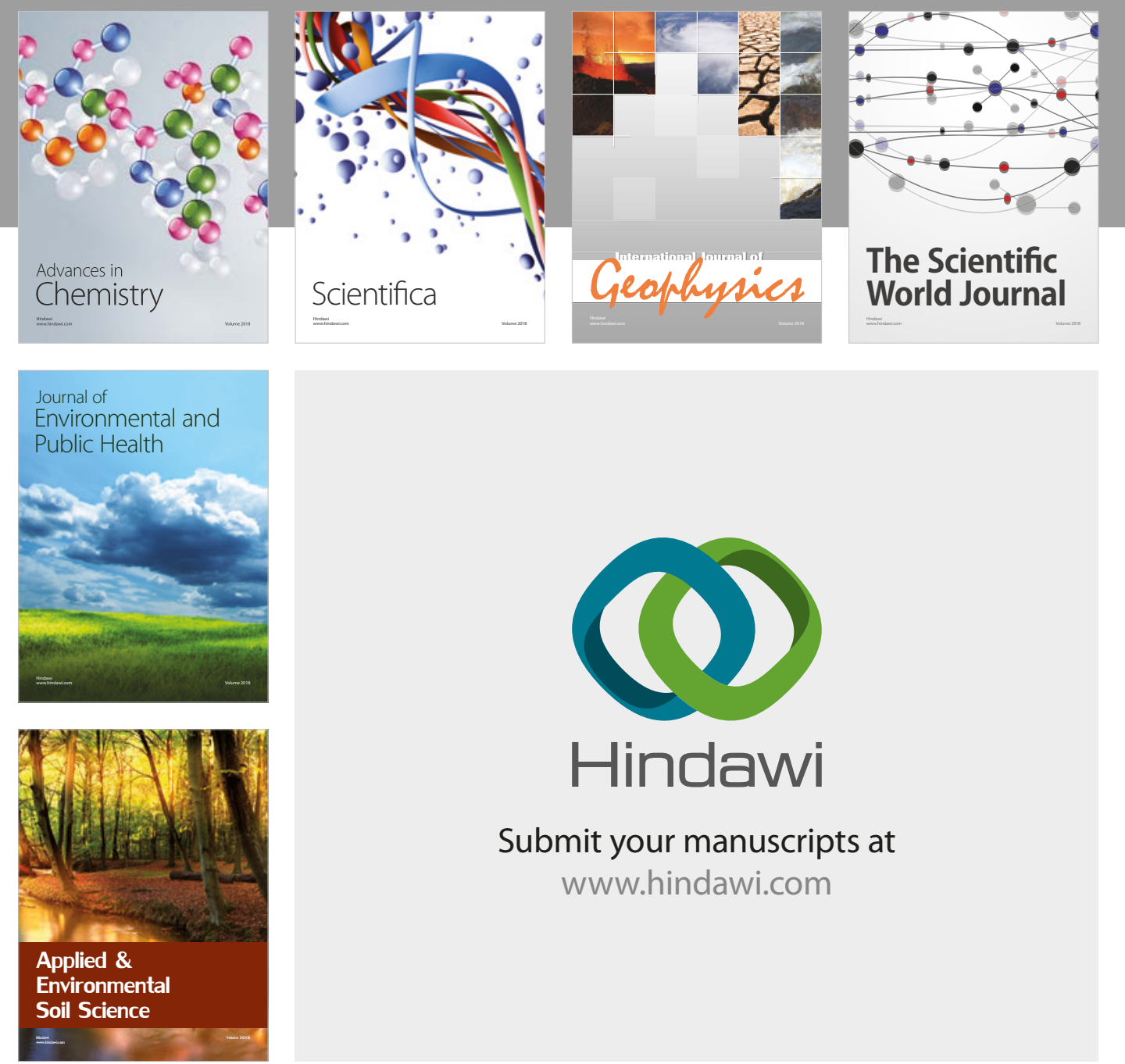

The Scientific

\section{World Journal}
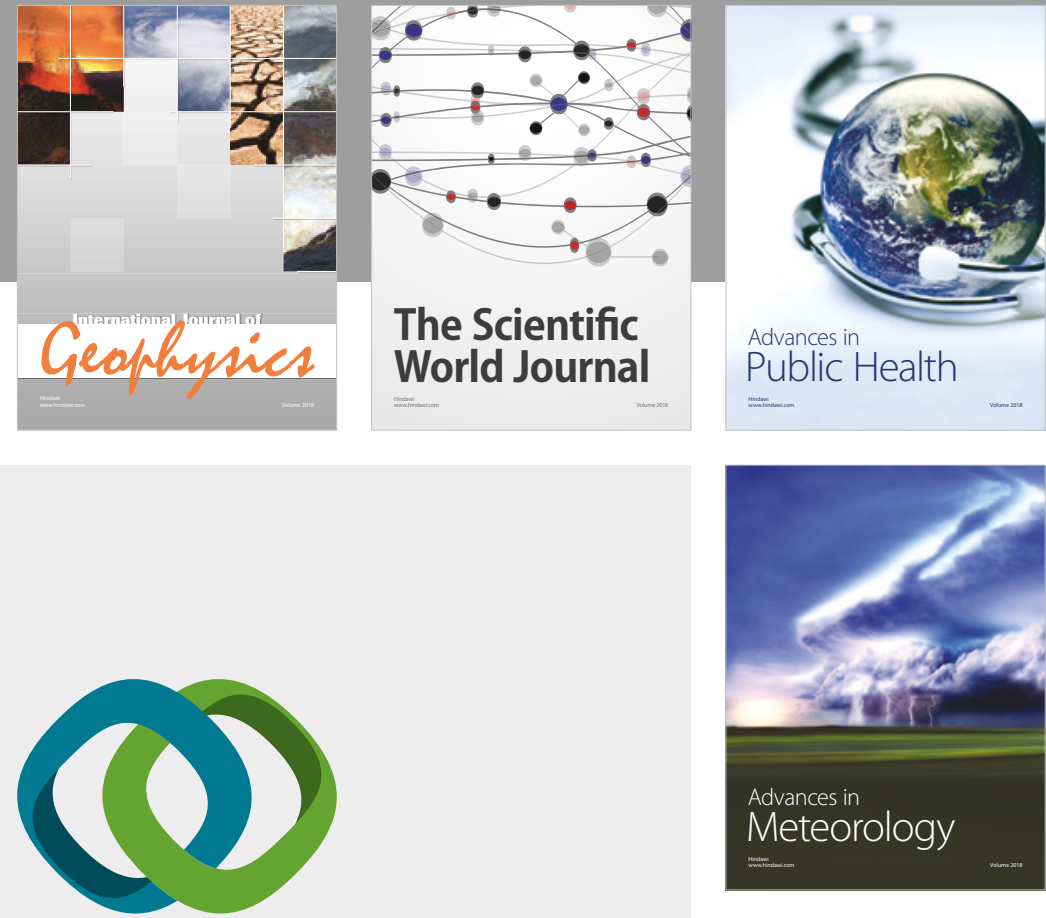

Advan

Public Health

\section{Hindawi}

Submit your manuscripts at

www.hindawi.com
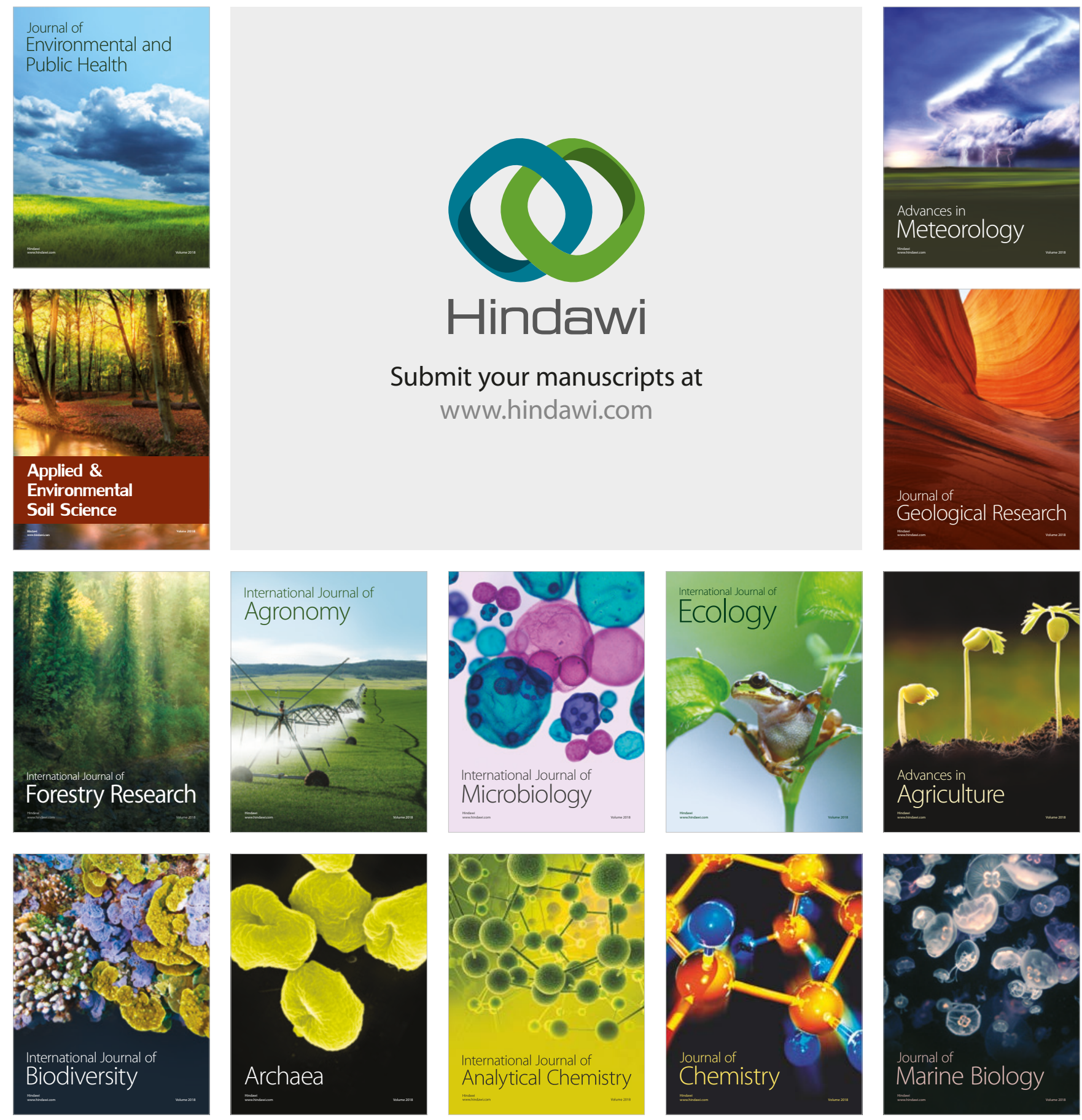